\title{
Mapping the human genetic architecture of COVID-19: an update
}

COVID-19 Host Genetics Initiative

\section{Abstract}

The Coronavirus Disease 2019 (COVID-19) pandemic continues to pose a major public health threat especially in countries with low vaccination rates. To better understand the biological underpinnings of SARS-CoV-2 infection and COVID-19 severity we formed the COVID19 Host Genetics Initiative. Here we present GWAS meta-analysis of up to 125,584 cases and over 2.5 million controls across 60 studies from 25 countries, adding 11 new genome-wide significant loci compared to those previously identified. Genes in novel loci include SFTPD, MUC5B and ACE2, reveal compelling insights regarding disease susceptibility and severity.

\section{Main text}

The Coronavirus Disease 2019 (COVID-19) pandemic continues to pose a major public health threat especially in countries with low vaccination rates. To better understand the biological underpinnings of SARS-CoV-2 infection and COVID-19 severity we formed the COVID19 Host Genetics Initiative. Here we present GWAS meta-analysis of up to 125,584 cases and over 2.5 million controls across 60 studies from 25 countries, adding 11 new genome-wide significant loci compared to those previously identified ${ }^{1}$. Genes in novel loci include SFTPD, MUC5B and $A C E 2$, reveal compelling insights regarding disease susceptibility and severity.

\section{Additional genomic regions identified for COVID-19 severity and SARS-CoV-2 infection}

Here we present meta-analyses bringing together 60 studies from 25 countries (Figure 1;

Supplementary Table 1) for three COVID-19 related phenotypes: (1) individuals critically ill with COVID-19 based on either requiring respiratory support in hospital or who died as a consequence of the disease $(9,376$ cases - of which 3,197 new in this data release - and 
medRxiv preprint doi: https://doi.org/10.1101/2021.11.08.21265944; this version posted February 9, 2022. The copyright holder for this preprint (which was not certified by peer review) is the author/funder, who has granted medRxiv a license to display the preprint in perpetuity.

It is made available under a CC-BY-NC-ND 4.0 International license .

$1,776,645$ controls), (2) cases with moderate or severe COVID-19 defined as those hospitalized due to symptoms associated with the infection (25,027 cases - 11,386 new - and 2,836,272 controls), and (3) all cases with reported SARS-CoV-2 infection regardless of symptoms $(125,584$ cases - 76,022 new - and 2,575,347 controls). An overview of the study design is provided in Supplementary Figure 1. We found a total of 23 genome-wide significant loci $(\mathrm{P}<$ $\left.5 \times 10^{-8}\right)$ of which 20 loci remain significant after multiple testing corrections $\left(P<1.67 \times 10^{-8}\right)$ to account for the number of phenotypes examined (Figure 2; Supplementary Figure 2;

Supplementary Table 2). We compared the effects of these loci between the previous 1 and the current analysis and found that only one locus did not replicate (rs72711165). All the other loci showed the expected increase in statistical significance (Supplementary Figure 3).

\section{Heterogeneity in genetic effects across phenotypes, studies, and ancestry groups}

Across the genome-wide significant loci, we observed clear patterns of association to the different phenotypes under study. Thus, we developed a two-class Bayesian model for classifying loci based on the patterns of association across the two better-powered phenotypes (COVID-19 hospitalization and SARS-CoV-2 reported infection). Intuitively, loci that are associated to susceptibility will also be associated to severity as to develop COVID-19, SARSCoV-2 infection needs to first occur. In contrast, those genetic effects that solely modify the course of illness should be associated to severity to illness and not show any association to reported infection except via preferential ascertainment of hospitalized cases in a cohort (Supplementary Methods). We identified 16 loci that are substantially more likely (> 99\% posterior probability) to impact the risk of COVID-19 hospitalization and 7 loci that clearly influence susceptibility to SARS-CoV-2 infection (Supplementary Table 3; Supplementary Figure 4).

We observed that several loci had a significant heterogeneous effect across studies (6/23 loci with P-value for heterogeneity $<2.2 \times 10^{-3}$; Supplementary Table 2). Thanks to an increased diversity in our study population (Supplementary Figure 5) we were able to explore if such heterogeneity was due to effect differences across continental ancestry groups. Only one locus (FOXP4) showed a significantly different effect across ancestries ( $\mathrm{P}$-value heterogeneity $<7 \times 10^{-}$ 5; Supplementary Table 4 and Supplementary Figure 6), though even at this locus all ancestry groups showed a positive effect estimate. This confirms that factors related to between-study heterogeneity (e.g., variable definition of COVID-19 severity due to different thresholds for testing, hospitalization, and patient recruitment) rather than differences across ancestries are a more likely explanation for the observed heterogeneity in the effect sizes across studies.

\section{Biological insights from novel loci}


medRxiv preprint doi: https://doi.org/10.1101/2021.11.08.21265944; this version posted February 9, 2022. The copyright holder for this preprint (which was not certified by peer review) is the author/funder, who has granted medRxiv a license to display the preprint in perpetuity. It is made available under a CC-BY-NC-ND 4.0 International license .

For the 23 genome-wide significant loci, we explored candidate causal genes and performed a phenome-wide association study to better understand their potential biological mechanisms (Supplementary Table 2,5,6; Supplementary Figure 7). Several of these loci with prior and direct connections to lung disease and SARS-CoV-2 infection mechanism are highlighted here.

Several loci involved in COVID-19 severity implicate lung surfactant biology. A missense variant rs721917:A>G (p.Met31Thr) in SFTPD (10q22.3) confers risk for hospitalization (OR [95\% confidence interval $\left.[\mathrm{Cl}]=1.06[1.04,1.08] ; P=1.7 \times 10^{-8}\right)$ and has been previously associated with increased risk of chronic obstructive pulmonary disease ${ }^{2}\left(\mathrm{OR}=1.08 ; P=2.0 \times 10^{-8}\right)$, and decreased lung function $^{3}$ (FEV1/FVC; $\beta=-0.019 ; P=2.0 \times 10^{-15}$ ). SFTPD encodes the surfactant protein D (SP-D) that participates in innate immune response, protecting the lungs against inhaled microorganisms. The recombinant fragment of SP-D binds to the S1 spike protein of the SARS-CoV-2 and potentially inhibits binding to ACE2 receptor and SARS-CoV-2 infection ${ }^{4}$. Another missense variant rs117169628:G>A (p.Pro256Leu) in SLC22A31 (16q24.3) also conferring risk for hospitalization $\left(\mathrm{OR}[95 \% \mathrm{Cl}]=1.09[1.06,1.13] ; P=2.6 \times 10^{-8}\right)$. $S L C 22 A 31$ belongs to the family of solute carrier proteins that facilitate transport across membranes ${ }^{5}$ and is co-regulated with other surfactant proteins ${ }^{6}$.

We found a variant rs35705950:G>T located in the promoter of MUC5B (11p15.5) to be protective against hospitalization (OR $\left.[95 \% \mathrm{Cl}]=0.83[0.86,0.93] ; P=6.5 \times 10^{-9}\right)$. This wellstudied promoter variant increases expression of MUC5B in lung in GTEx $\left(P=6.7 \times 10^{-16}\right)$ and is the strongest known variant associated with increased risk of developing idiopathic pulmonary fibrosis (IPF) ${ }^{7,8}$, but also improves survival in IPF patients carrying this mutation ${ }^{9}$.

Finally, we identified rs190509934:T>C, located 69 bp upstream of ACE2 (Xp22.2) to be associated with decreased susceptibility risk (OR $\left.[95 \% \mathrm{Cl}]=0.69[0.63,0.75] ; P=3.6 \times 10^{-18}\right)$. ACE2 is the SARS-CoV-2 receptor and functionally interacts with SLC6A19 and SLC6A20 ${ }^{10}$, one of which also showed a significant association with susceptibility (rs73062389:G $>A$ at SLC6A20; OR $\left.[95 \% \mathrm{Cl}]=1.18[1.16,1.20] ; P=2.5 \times 10^{-74}\right)$. Notably, rs 190509934 is 10 times more common in South Asians (MAF $=0.027$ ) than in Europeans (MAF $=0.0024$ ), demonstrating the importance of diversity for variant discovery. Recent results have shown that rs 190509934:T>C variant lowers ACE2 expression, which in turn confers protection from SARSCoV-2 infection ${ }^{11}$.

We applied Mendelian randomization to infer potential causal relationships between COVID-19 related phenotypes and their genetically correlated traits (Supplementary Methods;

Supplementary Tables 7,8,9; Supplementary Figure 8). A novel causal association was observed between genetic liability to type II diabetes (T2D) and SARS-CoV-2 reported infection $(\mathrm{OR}[95 \% \mathrm{Cl}]=1.02[1.01,1.03] ; \mathrm{P}=1.6 \times 10-3)$, and COVID-19 hospitalization $(\mathrm{OR}[95 \% \mathrm{Cl}]=$ $1.06[1.03,1.1] ; P=1.4 \times 10-4)$. Multivariable $M R(M V M R)$ was used to estimate the direct effect of liability to T2D on COVID-19-related phenotypes that was not mediated via BMI. This analysis indicated that the observed causal association of liability to T2D on COVID-19 phenotypes is mediated by BMI (Supplementary Table 10). 
medRxiv preprint doi: https://doi.org/10.1101/2021.11.08.21265944; this version posted February 9, 2022. The copyright holder for this preprint (which was not certified by peer review) is the author/funder, who has granted medRxiv a license to display the preprint in perpetuity. It is made available under a CC-BY-NC-ND 4.0 International license .

\section{Discussion}

We have substantially expanded the genetic analysis of SARS-CoV-2 infection and COVID-19 severity by doubling the case size, identifying 11 novel loci. We developed a new approach to systematically assign the 23 discovered loci to either disease susceptibility ( 7 loci) or disease severity (16 loci). While distinguishing the two phenotypes is challenging because progression to a severe form of the disease requires susceptibility to infection in the first place, it is now evident that the genetic mechanisms involved in these two aspects of the disease can be differentiated. Among the new loci associated with disease susceptibility, ACE2 represents an expected, yet interesting finding. MUC5B, SFTPD, and SLC22A31 are the three most interesting novel loci associated with COVID-19 severity. Their relationship with lung function and lung diseases is consistent with loci previously associated with disease severity. The SPs secreted by alveolar cells, representing an emerging biological mechanism, maintains healthy lung function and facilitates the clearance of pathogens ${ }^{12}$. The protective effect of the MUC5B variant is unexpected given the otherwise risk-increasing, concordant effect between IPF and COVID-19 observed for other variants ${ }^{8}$. Nonetheless, this result aligns with the MUC5B promoter variant association that shows a twofold higher survival rate among IPF patients ${ }^{9}$. In mice, Muc5b appears essential for effective mucociliary clearance and for controlling infection ${ }^{13}$ supporting therapies to control mucin secretion to be of potential benefit in COVID-19 patients.

Expanding genomic research to include participants from around the world enabled us to test if the effect of COVID-19related genetic variants was markedly different across ancestry groups. We didn't detect obvious heterogeneity between ancestry groups, and we attribute the observed heterogeneity in the effect of COVID-19 related genetic variants to the diverse inclusion criteria across studies in terms of COVID-19 severity. However, we also note that ascertainment differences across studies might mask true underlying differences in effect sizes between ancestry groups.

The novel biological insights gained by this expansion of the COVID-19 Host Genetic Initiative showed that increasing sample size and diversity remain a fruitful activity to better understand the human genetic architecture of COVID-19.

\section{Data Availability}

Summary statistics generated by COVID-19 HGI are available at https://www.covid19hg.org/results/r6/ . The analyses described here utilize the freeze 6 data. COVID-19 HGI continues to regularly release new data freezes. Summary statistics for nonEuropean ancestry samples are not currently available due to the small individual sample sizes of these groups, but results for 23 loci lead variants are reported in Supplementary Table 3. Individual level data can be requested directly from contributing studies, listed in Supplementary Table 1. We used publicly available data from GTEx (https://gtexportal.org/home/), the Neale lab (http://www.nealelab.is/uk-biobank/), Finucane lab (https://www.finucanelab.org), FinnGen Freeze 4 
medRxiv preprint doi: https://doi.org/10.1101/2021.11.08.21265944; this version posted February 9, 2022. The copyright holder for this preprint (which was not certified by peer review) is the author/funder, who has granted medRxiv a license to display the preprint in perpetuity.

It is made available under a CC-BY-NC-ND 4.0 International license .

cohort (https://www.finngen.fi/en/access_results), and eQTL catalogue release 3 (http://www.ebi.ac.uk/eqtl/).

\section{Code Availability}

The code for summary statistics lift-over, the projection PCA pipeline including precomputed loadings and meta-analyses are available on GitHub (https://github.com/covid19-hg/) and the code for the Mendelian randomization and genetic correlation pipeline is available on GitHub at https://github.com/marcoralab/MRcovid. Code for implementing the MVMR analysis is available at: https://github.com/marcoralab/multivariate_MR ; and subtype analyses at:

https://github.com/mjpirinen/covid19-hgi_subtypes

\section{Competing Interests}

A full list of competing interests is supplied as Supplementary Table 11.

\section{Author contributions}

Detailed author contributions are integrated in the authorship list.

\section{References}

1. COVID-19 Host Genetics Initiative. Mapping the human genetic architecture of COVID-19. Nature (2021). doi:10.1038/s41586-021-03767-x

2. Hobbs, B. D. et al. Genetic loci associated with chronic obstructive pulmonary disease overlap with loci for lung function and pulmonary fibrosis. Nat. Genet. 49, 426-432 (2017).

3. Shrine, N. et al. New genetic signals for lung function highlight pathways and chronic obstructive pulmonary disease associations across multiple ancestries. Nat. Genet. 51, 481-493 (2019).

4. Hsieh, M.-H. et al. Human Surfactant Protein D Binds Spike Protein and Acts as an Entry Inhibitor of SARS-CoV-2 Pseudotyped Viral Particles. Front. Immunol. 12, 641360 (2021).

5. Hediger, M. A. et al. The ABCs of solute carriers: physiological, pathological and therapeutic implications of human membrane transport proteinsIntroduction. Pflugers Arch. 447, 465-468 (2004).

6. Deelen, P. et al. Improving the diagnostic yield of exome- sequencing by predicting genephenotype associations using large-scale gene expression analysis. Nat. Commun. 10, 2837 (2019).

7. Seibold, M. A. et al. A common MUC5B promoter polymorphism and pulmonary fibrosis. $N$. Engl. J. Med. 364, 1503-1512 (2011).

8. Fadista, J. et al. Shared genetic etiology between idiopathic pulmonary fibrosis and COVID19 severity. EBioMedicine 65, 103277 (2021).

9. Peljto, A. L. et al. Association between the MUC5B promoter polymorphism and survival in patients with idiopathic pulmonary fibrosis. JAMA 309, 2232-2239 (2013).

10. Vuille-Dit-Bille, R. N. et al. Human intestine luminal ACE2 and amino acid transporter expression increased by ACE-inhibitors. Amino Acids 47, 693-705 (2014). 
medRxiv preprint doi: https://doi.org/10.1101/2021.11.08.21265944; this version posted February 9, 2022. The copyright holder for this preprint (which was not certified by peer review) is the author/funder, who has granted medRxiv a license to display the preprint in perpetuity. It is made available under a CC-BY-NC-ND 4.0 International license .

11. Horowitz, J. E. et al. Common genetic variants identify targets for COVID-19 and individuals at high risk of severe disease. medRxiv (2021). doi:10.1101/2020.12.14.20248176

12. Wright, J. R. Immunoregulatory functions of surfactant proteins. Nat. Rev. Immunol. 5, 5868 (2005).

13. Roy, M. G. et al. Muc5b is required for airway defence. Nature 505, 412-416 (2014).

\section{Figure Legends}

Figure 1 | Panel A: Geographical overview of the contributing studies to the COVID-19 HGI and composition by major ancestry groups. Populations are defined as Middle Eastern (MID), South Asian (SAS), East Asian (EAS), African (AFR), Admixed American (AMR), European (EUR). Panel B. Principal components analysis highlights the population structure and the sample ancestry of the individuals participating to the COVID-19 HGI. This figure is an updated version of the figure published by the COVID-19 Host Genetics Initiative ${ }^{1}$.

Figure 2 | Genome-wide association results for COVID-19. A. Top panel shows results of genome-wide association study of hospitalized COVID-19 ( $n=25,027$ cases and $n=2,836,272$ controls), and bottom panel the results of reported SARS-CoV-2 infection ( $n=125,584$ cases and $\mathrm{n}=2,575,347$ controls). Loci highlighted in yellow (top panel) represent regions associated with severity of COVID-19 manifestation. Loci highlighted in green (bottom panel) are regions associated with susceptibility to SARS-CoV-2 infection. Lead variants for the loci newly identified in this data release are annotated with their respective rsID. B. Results of gene prioritization using different evidence measures of gene annotation. Genes in linkage disequilibrium (LD) region, genes with coding variants and eGenes (fine-mapped cis-eQTL variant PIP > 0.1 in GTEx Lung) are annotated if in LD with a COVID-19 lead variant ( $\mathrm{r} 2>0.6$ ). V2G: Highest gene prioritized by OpenTargetGenetics' V2G score. The * indicates SARS-CoV-2 infection susceptibility and + indicates COVID-19 severity. The transparent loci were reported in the previous freeze (data release 5), and loci in bright blue were identified in the current freeze (data release 6). This figure is an updated version of the figure published by the COVID-19 Host Genetics Initiative ${ }^{1}$. 


\section{COVID-19 Host Genetics Initiative}

\section{Leadership}

\section{Leadership}

Gita A. Pathak ${ }^{1}$, Juha Karjalainen², Christine Stevens ${ }^{3}$, Benjamin M Neale ${ }^{4}$, Mark Daly ${ }^{2,3,5}$, Andrea Ganna ${ }^{2,3,5}$

\section{Writing group}

\section{Writing group lead}

Gita A. Pathak ${ }^{1}$, Shea J. Andrews ${ }^{6}$, Masahiro Kanai ${ }^{3}$, Mattia Cordioli ${ }^{7}$, Andrea Ganna ${ }^{2,3,5}$

\section{Analysis group}

Manuscript analyses team lead

Juha Karjalainen ${ }^{2}$

Manuscript analyses team members: phewas

Gita A. Pathak ${ }^{1}$, Renato Polimanti ${ }^{1}$

Manuscript analyses team members: mendelian randomization Shea J. Andrews ${ }^{6}$, Nadia Harerimana ${ }^{8}$

Manuscript analyses team members: methods development Mattia Cordioli ${ }^{7}$, Matti Pirinen $^{7}$

Manuscript analyses team members: PC projection, gene prioritization Masahiro Kanai $^{3}$

\section{Project management group}


Project management lead

Christine Stevens ${ }^{3}$,

Rachel G. Liao ${ }^{3}$

Project managment support

Karolina Chwialkowska ${ }^{9}$,

Amy Trankiem ${ }^{3}$,

Mary K. Balaconis ${ }^{3}$

\title{
Website development
}

Website development

Huy Nguyen ${ }^{3}$,

Matthew Solomonson ${ }^{3}$

\section{Scientific communication group}

Scientific communication lead

Kumar Veerapen ${ }^{3}$,

Brooke Wolford $^{10}$

\section{AncestryDNA COVID-19 Research Study}

\author{
Analysis Team Lead \\ Genevieve Roberts ${ }^{11}$ \\ Data Collection Lead \\ Danny Park ${ }^{11}$
}

\section{Admin Team Lead}

Catherine A. Ball ${ }^{11}$

Analysis Team Member

Marie Coignet ${ }^{11}$,

Shannon McCurdy ${ }^{11}$,

Spencer Knight ${ }^{11}$,

Raghavendran Partha ${ }^{11}$,

Brooke Rhead ${ }^{11}$

Data Collection Member 


\author{
Miao Zhang ${ }^{11}$, \\ Nathan Berkowitz ${ }^{11}$, \\ Michael Gaddis ${ }^{11}$, \\ Keith Noto ${ }^{11}$, \\ Luong Ruiz ${ }^{11}$, \\ Milos Pavlovic ${ }^{11}$
}

\title{
Admin Team Member
}

Eurie L. Hong ${ }^{11}$,

Kristin Rand ${ }^{11}$,

Ahna Girshick ${ }^{11}$,

Harendra Guturu ${ }^{11}$,

Asher Haug Baltzell ${ }^{11}$

\section{BelCovid}

\author{
Analysis Team Lead \\ Mari E.K. Niemi ${ }^{12}$ \\ Data Collection Lead \\ Souad Rahmouni ${ }^{13}$, \\ Julien Guntz ${ }^{14}$
}

\section{Admin Team Lead}

Yves Beguin ${ }^{15}$

\section{Analysis Team Member}

Mattia Cordioli ${ }^{16}$,

Sara Pigazzini ${ }^{12}$,

Lindokuhle Nkambule ${ }^{17,18,19}$

\section{Data Collection Member}


Michel Georges ${ }^{13}$,

Michel Moutschen ${ }^{20,21}$,

Benoit Misset $^{20,21}$,

Gilles Darcis ${ }^{20,21}$,

Julien Guiot ${ }^{20,21}$,

Samira Azarzar ${ }^{20,21}$,

Stéphanie Gofflot ${ }^{15}$,

Sabine Claassen ${ }^{14}$,

Olivier Malaise ${ }^{20}$

Pascale Huynen ${ }^{20}$,

Christelle Meuris ${ }^{22}$,

Marie Thys ${ }^{22}$,

Jessica Jacques $^{22}$,

Philippe Léonard ${ }^{22}$,

Frederic Frippiat $^{22}$,

Jean-Baptiste Giot $^{22}$,

Anne-Sophie Sauvage ${ }^{22}$,

Christian Von Frenckell ${ }^{22}$,

Yasmine Belhaj ${ }^{13}$,

Bernard Lambermont ${ }^{22}$

\section{Biobanque Quebec COVID19}

\section{Analysis Team Lead}

Tomoko Nakanishi ${ }^{23,24,25,26}$

\section{Data Collection Lead}

David R. Morrison ${ }^{24}$

\section{Admin Team Lead}

Vincent Mooser ${ }^{27}$,

J. Brent Richards ${ }^{24,28,29}$

\section{Analysis Team Member}

Guillaume Butler-Laporte ${ }^{24,30}$, Vincenzo Forgetta ${ }^{24}$,

Rui $\mathrm{Li}^{27}$

\section{Data Collection Member}




\author{
Biswarup Ghosh ${ }^{24}$, \\ Laetitia Laurent $^{24}$, \\ Alexandre Belisle ${ }^{27}$, \\ Danielle Henry ${ }^{24}$, \\ Tala Abdullah ${ }^{24}$, \\ Olumide Adeleye ${ }^{24}$, \\ Noor Mamlouk ${ }^{24}$, \\ Nofar Kimchi ${ }^{24}$, \\ Zaman Afrasiabi ${ }^{24}$, \\ Nardin Rezk ${ }^{24}$, \\ Branka Vulesevic ${ }^{24}$, \\ Meriem Bouab ${ }^{24}$, \\ Charlotte Guzman ${ }^{24}$, \\ Louis Petitjean ${ }^{24}$, \\ Chris Tselios ${ }^{24}$, \\ Xiaoqing $\mathrm{Xue}^{24}$, \\ Jonathan Afilalo ${ }^{24}$, \\ Marc Afilalo ${ }^{31,32}$, \\ Maureen Oliveira $^{33}$, \\ Bluma Brenner ${ }^{34}$, \\ Nathalie Brassard ${ }^{35}$, \\ Madeleine Durand ${ }^{36,37}$ \\ Admin Team Member \\ Erwin Schurr ${ }^{38}$, \\ Pierre Lepage ${ }^{27}$, \\ Jiannis Ragoussis ${ }^{27}$, \\ Daniel Auld ${ }^{27}$, \\ Michaël Chassés ${ }^{37,39}$, \\ Daniel E. Kaufmann ${ }^{37,40}$, \\ G. Mark Lathrop ${ }^{27}$, \\ Darin Adra ${ }^{24}$
}

\title{
CCHC COVID-19 GAWS
}

Analysis Team Lead

Caroline Hayward ${ }^{41}$,

Joseph T Glessner ${ }^{42,43}$, Douglas M Shaw ${ }^{44}$

\section{Data Collection Lead}

Archie Campbell ${ }^{45}$, Marcela Morris ${ }^{46}$

\section{Admin Team Lead}


Hakon Hakonarson ${ }^{42,43}$,

David J Porteous ${ }^{47}$,

Jennifer Below ${ }^{44}$

\section{Analysis Team Member}

Anne Richmond ${ }^{41}$,

Xiao Chang ${ }^{42}$,

Hannah Polikowski ${ }^{44}$,

Petty E Lauren ${ }^{44}$,

Hung-Hsin Chen ${ }^{44}$,

Zhu Wanying ${ }^{44}$

Data Collection Member

Chloe Fawns-Ritchie ${ }^{48}$

Admin Team Member

Kari North ${ }^{49}$,

Joseph B McCormick ${ }^{46}$

\section{CHOP_CAG}

\section{Data Collection Member}

Xiao Chang ${ }^{50}$,

Joseph R Glessner ${ }^{51,52}$,

Hakon Hakonarson ${ }^{51,53,54}$

\section{Colorado Center for Personalized Medicine (CCPM)}

Analysis Team Lead

Christopher R Gignoux ${ }^{55}$

\section{Data Collection Lead}

Stephen J Wicks ${ }^{55}$,

Kristy Crooks ${ }^{55}$

\section{Admin Team Lead}

Kathleen C Barnes ${ }^{55}$

Analysis Team Member

Michelle Daya ${ }^{55}$,

Jonathan Shortt ${ }^{55}$,

Nicholas Rafaels ${ }^{55}$,

Sameer Chavan ${ }^{55}$

\section{Coronagenes}




\section{Analysis Team Lead}

Paul RHJ Timmers ${ }^{56,57}$, James F Wilson ${ }^{56,57}$, Albert Tenesa ${ }^{56,58}$

\section{Admin Team Lead}

Shona M Kerr ${ }^{56}$

\section{Analysis Team Member \\ Kenton D'Mellow ${ }^{58}$}

\section{Egypt hgCOVID hub}

\section{Analysis Team Lead}

Mari E.K. Niemi ${ }^{12}$

\section{Data Collection Lead}

Doaa Shahin ${ }^{59}$,

Yasser M. El-Sherbiny ${ }^{59,60}$

\section{Admin Team Lead}

Kathrin Aprile von Hohenstaufen ${ }^{61}$,

Ali Sobh ${ }^{62}$,

Madonna M. Eltoukhy ${ }^{63}$

\section{Analysis Team Member}

Mattia Cordioli ${ }^{7}$,

Lindokuhle Nkambul ${ }^{17,64}$

\section{Data Collection Member}

Tamer A. Elhadidy ${ }^{65}$,

Mohamed S. Abd Elghafar ${ }^{66}$,

Jehan J. El-Jawhari ${ }^{59,60}$,

Attia A.S. Mohamed ${ }^{63}$,

Marwa H. Elnagdy ${ }^{67}$,

Amr Samir ${ }^{68}$,

Mahmoud Abdel-Aziz ${ }^{69}$,

Walid T. Khafaga ${ }^{70}$,

Walaa M. El-Lawaty ${ }^{71}$,

Mohamed S. Torky ${ }^{71}$,

Mohamed R. El-shanshory ${ }^{72}$

Admin Team Member 
Amr M. Yassen ${ }^{73}$,

Mohamed A.F. Hegazy ${ }^{68}$,

Kamal Okasha ${ }^{74}$,

Mohammed A. Eid ${ }^{75}$,

Hanteera S. Moahmed ${ }^{71}$

\title{
EraCORE
}

\section{Analysis Team Lead}

Carolina Medina-Gomez ${ }^{76}$

\section{Data Collection Lead}

M. Arfan Ikram ${ }^{77}$

\section{Admin Team Lead}

Andre G Uitterlinden ${ }^{76,77}$

\section{Estonian Biobank}

\author{
Analysis Team Lead \\ Reedik Mägi $^{78}$
}

Data Collection Lead

Lili Milani ${ }^{78}$

\section{Admin Team Lead}

Andres Metspalu ${ }^{78}$

\section{Analysis Team Member}

Triin Laisk $^{78}$,

Kristi Läll ${ }^{78}$,

Maarja Lepamets $^{78}$

\section{Data Collection Member}




\author{
Tõnu Esko ${ }^{78}$, \\ Ene Reimann ${ }^{78}$, \\ Paul Naaber ${ }^{79}$, \\ Edward Laane ${ }^{80,81}$, \\ Jaana Pesukova ${ }^{80}$, \\ Pärt Peterson ${ }^{82}$, \\ Kai Kisand ${ }^{83}$, \\ Jekaterina Tabri ${ }^{84}$, \\ Raili Allos ${ }^{84}$, \\ Kati Hensen ${ }^{84}$, \\ Joel Starkopf ${ }^{85}$, \\ Inge Ringmets ${ }^{86}$, \\ Anu Tamm ${ }^{87}$, \\ Anne Kallaste ${ }^{87}$
}

\title{
Admin Team Member
}

Helene Alavere ${ }^{78}$,

Kristjan Metsalu ${ }^{78}$,

Mairo Puusepp ${ }^{78}$

\section{EXCEED}

\section{Analysis Team Lead \\ Chiara Batini $^{88}$}

Data Collection Lead

Martin D Tobin 88,89

Admin Team Lead

Laura D Venn ${ }^{88}$

\section{Analysis Team Member}

Paul H Lee ${ }^{88}$,

Nick Shrine ${ }^{88}$,

Alexander T Williams ${ }^{88}$

\section{Data Collection Member}


Anna L Guyatt ${ }^{88}$, Catherine John ${ }^{88}$,

Richard J Packer ${ }^{88}$, Altaf $\mathrm{Ali}^{88}$, Robert C Free ${ }^{90}$, Xueyang Wang ${ }^{88}$, Louise V Wain ${ }^{88,89}$, Edward J Hollox ${ }^{91}$

Admin Team Member Catherine $\mathrm{E} \mathrm{Bee}{ }^{88}$, Emma L Adams ${ }^{88}$

\section{FinnGen}

Admin Team Lead

FinnGen $^{92}$

Analysis Team Member

Samuli Ripatti ${ }^{3,93,94,95}$,

Sanni Ruotsalainen ${ }^{93}$

Data Collection Member

Kati Kristiansson ${ }^{96}$,

Sami Koskelainen ${ }^{96}$,

Markus Perola ${ }^{96,97}$,

Kati Donner ${ }^{7}$,

Katja Kivinen ${ }^{7}$,

Aarno Palotie $^{7}$

Admin Team Member

Mari Kaunisto $^{7}$

\section{Functional Host Genomics in Infectious Diseases (FHoGID)}

Analysis Team Lead

Carlo Rivolta ${ }^{98,99}$

\section{Data Collection Lead}


Pierre-Yves Bochud ${ }^{100}$,

Stéphanie Bibert ${ }^{101}$,

Noémie Boillat ${ }^{100}$,

Semira Gonseth Nussle ${ }^{102}$,

Werner Albrich ${ }^{103}$

\section{Analysis Team Member}

Mathieu Quinodoz ${ }^{98,99}$,

Dhryata Kamdar ${ }^{98,99}$

\section{Data Collection Member}

Noémie Suh ${ }^{104}$,

Dionysios Neofytos ${ }^{105}$,

Véronique Erard $^{106}$,

Cathy Voide ${ }^{107}$,

FHoGID $^{108}$,

RegCOVID ${ }^{109}$,

P-PredictUs ${ }^{110}$,

SeroCOVID ${ }^{111}$, CRiPSI ${ }^{112}$

\section{GCAT. Genomes For Life.}

\section{Analysis Team Lead \\ Rafael de $\mathrm{Cid}^{113}$}

\section{Data Collection Lead}

Anna Carreras ${ }^{113}$,

Victor Moreno ${ }^{114}$,

Manolis Kogevinas ${ }^{115,116,117,118}$

\section{Analysis Team Member}

Iván Galván-Femenía ${ }^{113}$, Natalia Blay ${ }^{113}$,

Xavier Farré ${ }^{113}$,

Lauro Sumoy ${ }^{113}$

\section{Data Collection Member}


medRxiv preprint doi: https://doi.org/10.1101/2021.11.08.21265944; this version posted February 9, 2022. The copyright holder for this preprint (which was not certified by peer review) is the author/funder, who has granted medRxiv a license to display the preprint in perpetuity. It is made available under a CC-BY-NC-ND 4.0 International license.

\author{
Beatriz Cortés ${ }^{113}$, \\ Josep Maria Mercader ${ }^{119}$, \\ Marta Guindo-Martinez ${ }^{120}$, \\ David Torrents ${ }^{120}$, \\ Judith Garcia-Aymerich ${ }^{115,117,118}$, \\ Gemma Castaño-Vinyals ${ }^{115,116,117,118}$, \\ Carlota Dobaño ${ }^{115,118}$
}

\title{
GEN-COVID Multicenter Study
}

\section{Analysis Team Lead}

Marco Gori ${ }^{121,122}$,

Mari E.K. Niemi ${ }^{12}$

\section{Data Collection Lead}




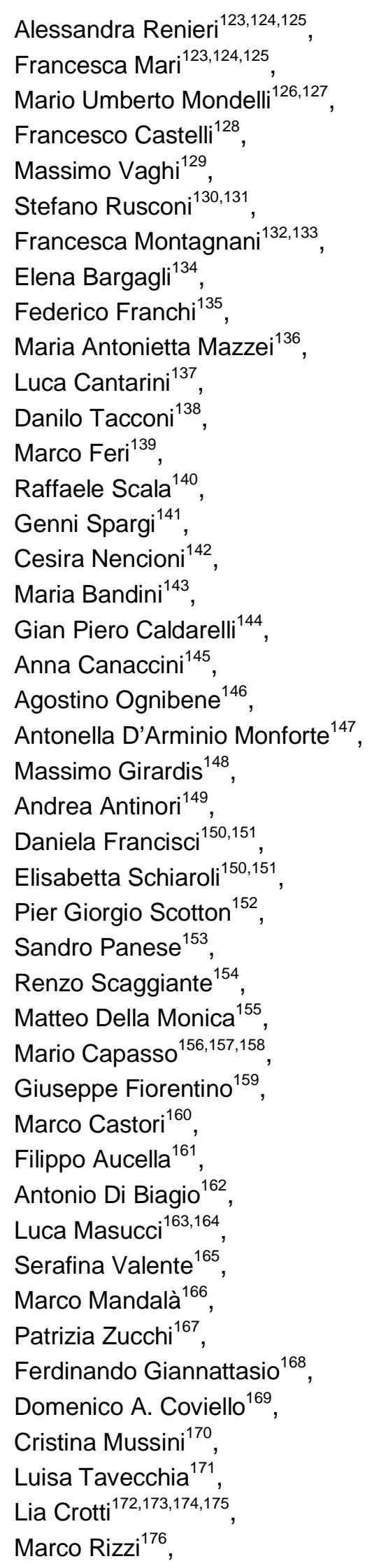




\author{
Maria Teresa La Rovere ${ }^{177}$, \\ Simona Sarzi-Braga ${ }^{178}$, \\ Maurizio Bussotti ${ }^{179}$, \\ Sabrina Ravaglia ${ }^{180}$, \\ Rosangela Artuso ${ }^{181}$, \\ Antonio Perrella ${ }^{182}$, \\ Davide Romani ${ }^{183}$, \\ Paola Bergomi ${ }^{184}$, \\ Emanuele Catena ${ }^{184}$, \\ Antonella Vincenti ${ }^{185}$, \\ Claudio Ferri ${ }^{186}$, \\ Davide Grassi ${ }^{186}$, \\ Gloria Pessina ${ }^{187}$, \\ Mario Tumbarello ${ }^{132,188}$, \\ Massimo Di Pietro ${ }^{189}$, \\ Ravaglia Sabrina ${ }^{190}$, \\ Sauro Luchi ${ }^{191}$
}

\title{
Admin Team Lead
}

Simone Furini ${ }^{125}$,

Simona Dei ${ }^{192}$

\section{Analysis Team Member}

Elisa Benetti ${ }^{125}$,

Nicola Picchiotti ${ }^{121,193}$,

Maurizio Sanarico ${ }^{194}$,

Stefano Ceri ${ }^{195}$,

Pietro Pinoli ${ }^{195}$,

Francesco Raimondi ${ }^{196}$,

Filippo Biscarini ${ }^{197}$,

Alessandra Stella $^{198}$,

Kristina Zguro ${ }^{199}$,

Katia Capitani ${ }^{132,200}$,

Mattia Cordioli ${ }^{16}$,

Sara Pigazzini ${ }^{12}$,

Mattia Cordioli ${ }^{16}$,

Sara Pigazzini ${ }^{12}$,

Lindokuhle Nkambule ${ }^{17,64}$,

Marco Tanfoni ${ }^{121}$

\section{Data Collection Member}




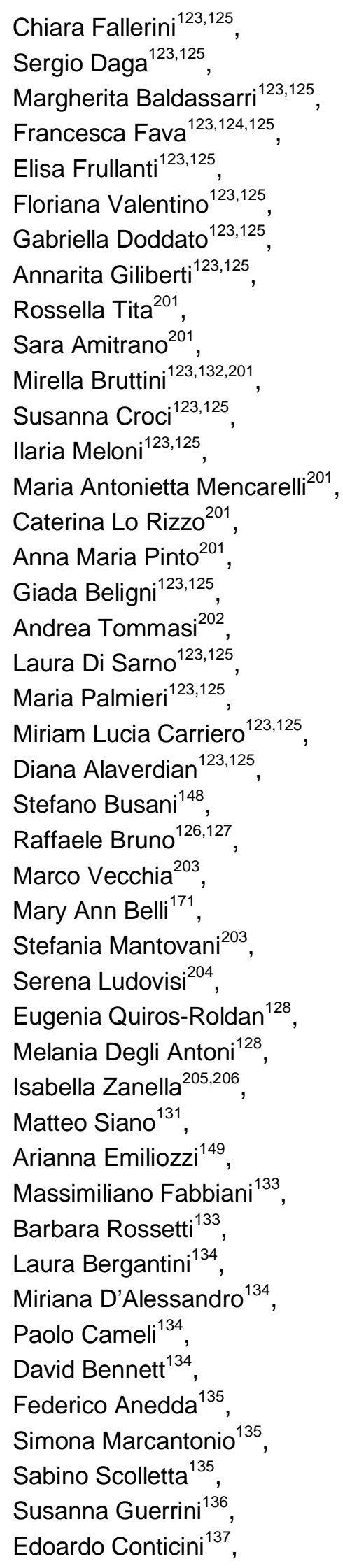




\author{
Bruno Frediani ${ }^{137}$, \\ Chiara Spertilli ${ }^{138}$, \\ Alice Donati ${ }^{139}$, \\ Luca Guidelli $^{140}$, \\ Marta Corridi ${ }^{141}$, \\ Leonardo Croci ${ }^{142}$, \\ Paolo Piacentini ${ }^{143}$, \\ Elena Desanctis ${ }^{143}$, \\ Silvia Cappelli ${ }^{143}$, \\ Agnese Verzuri ${ }^{145}$, \\ Valentina Anemoli ${ }^{145}$, \\ Alessandro Pancrazzi ${ }^{146}$, \\ Maria Lorubbio $^{146}$, \\ Federica Gaia Miraglia ${ }^{147}$, \\ Sophie Venturelli ${ }^{148}$ \\ Andrea Cossarizza ${ }^{207}$, \\ Alessandra Vergori ${ }^{149}$, \\ Arianna Gabrieli ${ }^{131}$, \\ Agostino Riva ${ }^{130,131}$, \\ Francesco Paciosi ${ }^{151}$, \\ Francesca Andretta ${ }^{152}$, \\ Francesca Gatti ${ }^{154}$, \\ Saverio Giuseppe Parisi ${ }^{208}$, \\ Stefano Baratti ${ }^{208}$, \\ Carmelo Piscopo ${ }^{155}$, \\ Roberta Russo 156,157, \\ Immacolata Andolfo ${ }^{156,157}$, \\ Achille Iolascon ${ }^{156,157}$, \\ Massimo Carella ${ }^{160}$, \\ Giuseppe Merla ${ }^{156,209}$, \\ Gabriella Maria Squeo ${ }^{209}$, \\ Pamela Raggi ${ }^{210}$, \\ Carmen Marciano ${ }^{210}$, \\ Rita Perna ${ }^{210}$, \\ Matteo Bassetti ${ }^{162,211}$, \\ Maurizio Sanguinetti ${ }^{163,164}$, \\ Alessia Giorli ${ }^{166}$, \\ Lorenzo Salerni $^{166}$, \\ Pierpaolo Parravicini ${ }^{167}$, \\ Elisabetta Menatti $^{212}$, \\ Tullio Trotta ${ }^{168}$, \\ Gabriella Coiro ${ }^{168}$, \\ Fabio Lena ${ }^{213}$, \\ Enrico Martinelli ${ }^{214}$,
}




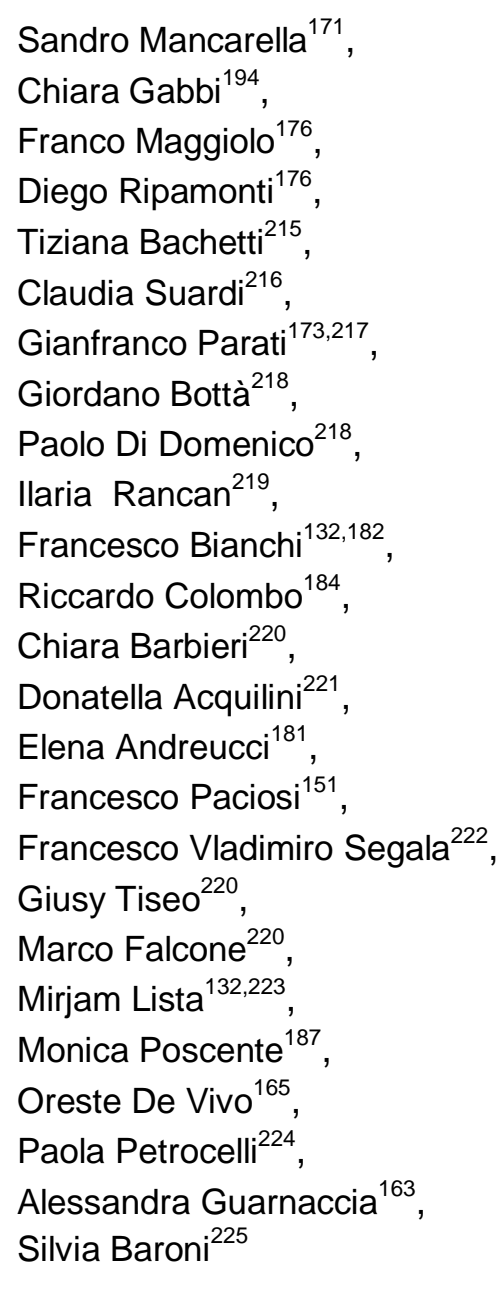

\section{Generation Scotland}

Analysis Team Lead

Caroline Hayward ${ }^{226}$

Data Collection Lead

David J Porteous ${ }^{227}$

Admin Team Lead

Chloe Fawns-Ritchie ${ }^{228}$

Analysis Team Member

Anne Richmond ${ }^{226}$

Data Collection Member

Archie Campbell ${ }^{227}$

\section{Genes \& Health}




\author{
Analysis Team Lead \\ David A van Heel ${ }^{229}$ \\ Data Collection Lead \\ Karen A Hunt ${ }^{229}$ \\ Admin Team Lead \\ Richard C Trembath ${ }^{230}$ \\ Analysis Team Member \\ Qin Qin Huang ${ }^{231}$, \\ Hilary C Martin ${ }^{231}$ \\ Data Collection Member \\ Dan Mason ${ }^{232}$, \\ Bhavi Trivedi ${ }^{233}$, \\ John Wright ${ }^{232}$

\section{Admin Team Member} \\ Sarah Finer ${ }^{234}$, \\ Genes \& Health Research Team ${ }^{235}$, \\ Christopher J Griffiths ${ }^{236}$
}

\title{
Genes for Good
}

Analysis Team Lead

Albert V Smith ${ }^{237}$

\section{Data Collection Member}

Andrew $\mathrm{P}$ Boughton ${ }^{237}$,

Kevin W. $\mathrm{Li}^{237}$,

Jonathon LeFaive ${ }^{237}$,

Aubrey Annis ${ }^{237}$

\section{Genome-wide assessment of the gene variants associated with severe COVID-19 phenotype in Iran}

Analysis Team Lead

Mari E.K. Niemi ${ }^{12}$,

Ahmadreza Niavarani ${ }^{238}$

Data Collection Lead 
Rasoul Aliannejad ${ }^{239}$

\section{Analysis Team Member \\ Mattia Cordioli ${ }^{7}$, \\ Lindokuhle Nkambul 17,64, \\ Bahareh Sharififard ${ }^{238}$}

\section{Data Collection Member}

Ali Amirsavadkouhi ${ }^{240}$,

Zeinab Naderpour ${ }^{239}$, Hengameh Ansari Tadi ${ }^{241}$,

Afshar Etemadi Aleagha ${ }^{242}$,

Saeideh Ahmadi ${ }^{243}$,

Seyed Behrooz Mohseni Moghaddam ${ }^{244}$,

Alireza Adamsara ${ }^{245}$,

Morteza Saeedi ${ }^{246}$,

Hamed Abdollahi ${ }^{247}$,

Abdolmajid Hosseini ${ }^{248}$

\section{Host genetic factors in COVID-19 patients in relation to disease susceptibility, disease severity and pharmacogenomics}

Analysis Team Lead

Pajaree Chariyavilaskul ${ }^{249,250}$

Data Collection Lead

Watsamon Jantarabenjakul ${ }^{251,252}$

\section{Admin Team Lead}

Nattiya Hirankarn ${ }^{253,254}$

\section{Analysis Team Member}

Monpat Chamnanphon ${ }^{255,256}$,

Thitima B. Suttichet ${ }^{255}$,

Vorasuk Shotelersuk ${ }^{257,258}$,

Monnat Pongpanich ${ }^{259,260}$,

Chureerat Phokaew ${ }^{258,261,262}$,

Wanna Chetruengchai ${ }^{258,262}$

\section{Data Collection Member}


Opass Putchareon ${ }^{251,263}$, Pattama Torvorapanit ${ }^{251,263}$, Thanyawee Puthanakit ${ }^{252,264}$, Pintip Suchartlikitwong ${ }^{264,265}$

Admin Team Member

Voraphoj Nilaratanakul ${ }^{266,267}$, Pimpayao Sodsai ${ }^{253,254}$

\section{HUNT}

\section{Analysis Team Lead}

Ben M Brumpton ${ }^{268,269,270}$

\section{Data Collection Lead}

Kristian Hveem 268,269 Cristen Willer $271,272,273$

\section{Analysis Team Member}

Brooke Wolford $^{271,272,273}$, Wei Zhou 274,275

\section{Data Collection Member}

Tormod Rogne 276,277,278,

Erik Solligard $^{276,278}$,

Bjørn Olav Åsvold ${ }^{268,269,270}$

\section{Lifelines}

Analysis Team Lead

Lude Franke ${ }^{279}$

\section{Data Collection Lead}

Marike Boezen ${ }^{280}$

\section{Analysis Team Member}

Patrick Deelen ${ }^{281}$,

Annique Claringbould ${ }^{279}$,

Esteban Lopera $^{279}$,

Robert Warmerdam ${ }^{279}$,

Judith. M. Vonk ${ }^{282}$,

Irene van Blokland ${ }^{279}$

\section{Data Collection Member}


Pauline Lanting ${ }^{283}$,

Anil P. S. Ori ${ }^{284,285}$

\title{
Mass General Brigham - Host Vulnerability to COVID-19
}

\author{
Analysis Team Lead \\ Yen-Chen Anne Feng ${ }^{286}$, \\ Josep Mercader 287,288 \\ Data Collection Lead \\ Scott T Weiss ${ }^{289}$, \\ Elizabeth W. Karlson ${ }^{290}$, \\ Jordan W. Smoller ${ }^{291}$, \\ Shawn N Murphy ${ }^{292}$, \\ James B. Meigs ${ }^{293}$, \\ Ann E. Woolley ${ }^{290}$ \\ Admin Team Lead \\ Robert C. Green ${ }^{294}$ \\ Data Collection Member \\ Emma F Perez ${ }^{295}$
}

\section{Michigan Genomics Initiative (MGI)}

Analysis Team Lead

Brooke Wolford $^{273}$

Admin Team Lead

Sebastian Zöllner ${ }^{237}$

Analysis Team Member Jiongming Wang ${ }^{237}$, Andrew Beck ${ }^{237}$

\section{Mount Sinai Health System COVID-19 Genomics Initiative}

Analysis Team Lead

Laura G. Sloofman 296,297,298

Data Collection Lead 


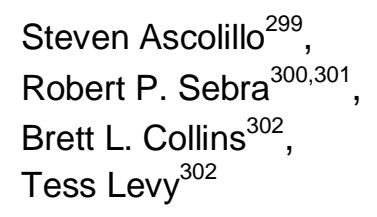

\section{Admin Team Lead}

Joseph D. Buxbaum ${ }^{302}$, Stuart C. Sealfon ${ }^{298}$

\section{Analysis Team Member}

Shea J. Andrews ${ }^{6}$,

Daniel M. Jordan ${ }^{303,304}$,

Ryan C. Thompson ${ }^{305,306,307}$, Kyle Gettler ${ }^{308}$,

Kumardeep Chaudhary ${ }^{304,309}$, Gillian M. Belbin ${ }^{310}$, Michael Preuss ${ }^{311,312}$,

Clive Hoggart ${ }^{298,313}$,

Sam Choi ${ }^{314}$,

Slayton J. Underwood ${ }^{298,315}$

\section{Data Collection Member}

Irene Salib ${ }^{300}$,

Bari Britvan ${ }^{302}$,

Katherine Keller ${ }^{302}$,

Lara Tang ${ }^{302}$,

Michael Peruggia ${ }^{302}$,

Liam L. Hiester ${ }^{302}$,

Kristi Niblo ${ }^{302}$,

Alexandra Aksentijevich ${ }^{302}$,

Alexander Labkowsky ${ }^{302}$,

Avromie Karp $^{302}$,

Menachem Zlatopolsky ${ }^{302}$,

Marissa Zyndorf ${ }^{300}$

\section{Admin Team Member}


Alexander W. Charney ${ }^{316}$,

Noam D. Beckmann ${ }^{299}$,

Eric E. Schadt ${ }^{300,301}$,

Noura S. Abul-Husn ${ }^{310}$,

Judy H. Cho ${ }^{304,309}$,

Yuval Itan 304,309 ,

Eimear E. Kenny ${ }^{310}$,

Ruth J.F. Loos ${ }^{311,312,317}$,

Girish N. Nadkarni ${ }^{311,318,319,320,321}$,

Ron Do ${ }^{304,309}$,

Paul O'Reilly ${ }^{314}$,

Laura M. Huckins ${ }^{322}$

\section{MyCode Health Initiative}

Analysis Team Lead

Manuel A.R. Ferreira ${ }^{323}$,

Goncalo R. Abecasis 323

Data Collection Lead

Joseph B. Leader ${ }^{324}$,

Michael N. Cantor ${ }^{323}$

\section{Admin Team Lead}

Anne E Justice ${ }^{325}$,

Dave J. Carey ${ }^{326}$

\section{Analysis Team Member}

Geetha Chittoor $^{325}$,

Navya Shilpa Josyula ${ }^{325}$,

Jack A. Kosmicki ${ }^{323}$,

Julie E Horowitz ${ }^{323}$,

Aris Baras ${ }^{323}$

Data Collection Member

Matthew C. Gass ${ }^{324}$,

Ashish Yadav ${ }^{323}$

Admin Team Member

Tooraj Mirshahi ${ }^{326}$

\section{Netherlands Twin Register}

\section{Analysis Team Lead}


Jouke Jan Hottenga ${ }^{327}$

Data Collection Lead

Meike Bartels ${ }^{327}$

Admin Team Lead

Eco (E.J.C.) de geus ${ }^{327}$

Analysis Team Member

Michel (M.G.) Nivard ${ }^{327}$

\section{Penn Medicine Biobank}

Analysis Team Lead

Anurag Verma ${ }^{328}$,

Marylyn D. Ritchie ${ }^{328}$

Admin Team Lead

Daniel Rader ${ }^{328}$

Analysis Team Member

Binglan $\mathrm{Li}^{329}$,

Shefali S Verma ${ }^{328}$,

Anastasia Lucas ${ }^{328}$,

Yuki Bradford ${ }^{328}$

\section{Saudi Human Genome Program- COVID19 : Host Genomic markers predicting the severity and suitability to COVID-19 in highly consanguineous population}

Analysis Team Lead

Malak Abedalthagafi ${ }^{330}$, Manal Alaamery ${ }^{331}$

\section{Data Collection Lead}

Abdulraheem Alshareef ${ }^{332}$, Mona Sawaji ${ }^{333}$

Admin Team Lead

Salam Massadeh ${ }^{331}$, Abdulaziz AIMalik ${ }^{334}$

Analysis Team Member 


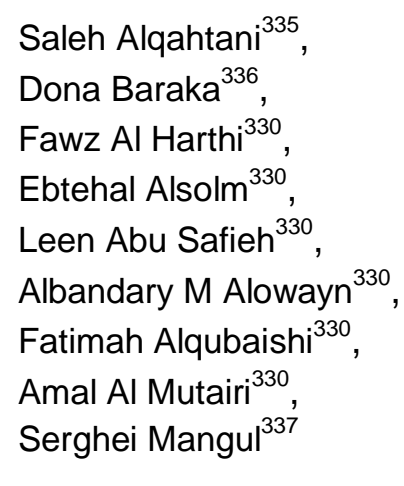

\section{Data Collection Member}

Mansour Almutairi ${ }^{331}$, Nora Aljawini ${ }^{338}$,

Nour Albesher ${ }^{338}$, Yaseen M Arabi ${ }^{339}$, Ebrahim S Mahmoud ${ }^{339}$, Amin K Khattab ${ }^{340}$, Roaa T Halawani ${ }^{340}$, Ziab Z Alahmadey ${ }^{340}$, Jehad K Albakri ${ }^{340}$, Walaa A Felemban ${ }^{340}$, Bandar A Suliman ${ }^{332}$, Rana Hasanato ${ }^{336}$, Laila Al-Awdah ${ }^{341}$, Jahad Alghamdi ${ }^{342}$, Deema AlZahrani ${ }^{343}$, Sameera AlJohani ${ }^{344}$, Hani Al-Afghani ${ }^{345}$, Nouf AIDhawi ${ }^{343}$, Hadeel AIBardis ${ }^{330}$, Sarah Alkwai ${ }^{338}$, Moneera Alswailm ${ }^{338}$, Faisal Almalki ${ }^{343}$, Maha Albeladi ${ }^{343}$, Iman Almohammed ${ }^{338}$, Eman Barhoush ${ }^{346}$, Anoud Albader ${ }^{343}$

\section{Admin Team Member}

Sara Alotaibi ${ }^{330}$, Bader Alghamdi $^{347}$, Junghyun Jung ${ }^{348}$, Mohammad S fawzy ${ }^{330}$

\section{Data collection Member}


May Alrashed ${ }^{349}$

\title{
The genetic predisposition to severe COVID-19
}

\author{
Analysis Team Lead \\ Mari E.K. Niemi ${ }^{12}$ \\ Data Collection Lead \\ Hugo Zeberg ${ }^{350,351}$
}

Analysis Team Member

Mattia Cordioli ${ }^{16}$,

Sara Pigazzini ${ }^{12}$,

Lindo Nkambul ${ }^{3,17,352}$

\section{Data Collection Member}

Robert Frithiof ${ }^{353}$,

Michael Hultström ${ }^{353,354}$,

Miklos Lipcsey ${ }^{353,355}$,

Nicolas Tardif ${ }^{356}$,

Olav Rooyackers ${ }^{356}$,

Jonathan Grip ${ }^{356}$,

Tomislav Maricic ${ }^{351}$

\section{The Norwegian Mother, Father and Child Cohort Study}

Analysis Team Lead

Øyvind Helgeland ${ }^{357}$

Data Collection Lead

Per Magnus ${ }^{358}$,

Lill-Iren S Trogstad ${ }^{359}$

Analysis Team Member

Yunsung Lee ${ }^{358}$

Admin Team Member

Jennifer R Harris ${ }^{357}$

\section{TwinsUK}

Analysis Team Lead

Massimo Mangino ${ }^{360,361}$

Data Collection Lead 
Tim D Spector ${ }^{360}$

Data Collection Member

Duncan Emma ${ }^{360}$

\title{
UK 100,000 Genomes Project (Genomics England)
}

\author{
Analysis Team Lead \\ Loukas Moutsianas ${ }^{362,363}$ \\ Data Collection Lead \\ Mark J Caulfield ${ }^{362,363,364,}$ \\ Richard H Scott ${ }^{362,365}$
}

Analysis Team Member

Athanasios Kousathanas ${ }^{366}$,

Dorota Pasko ${ }^{366}$,

Susan Walker ${ }^{366}$,

Alex Stuckey ${ }^{366}$,

Christopher A Odhams ${ }^{366}$,

Daniel Rhodes ${ }^{366}$

\section{Data Collection Member}

Tom Fowler ${ }^{366}$,

Augusto Rendon ${ }^{362,367}$,

Georgia Chan ${ }^{366}$,

Prabhu Arumugam ${ }^{366}$

\section{UK Biobank}

\section{Analysis Team Lead}

Tomoko Nakanishi ${ }^{368}$,

Konrad J. Karczewski ${ }^{5,19}$,

Alicia R. Martin ${ }^{5,19}$,

Daniel J Wilson ${ }^{369}$,

Chris C A Spencer ${ }^{370}$

\section{Data Collection Lead}

Derrick W Crook ${ }^{371}$,

David H Wyllie 371,372 ,

Anne Marie O'Connell ${ }^{373}$

\section{Admin Team Lead}


J. Brent Richards ${ }^{24,28,29}$

\section{Analysis Team Member}

Guillaume Butler-Laporte ${ }^{24,30}$, Vincenzo Forgetta ${ }^{24}$,

Elizabeth G. Atkinson ${ }^{5,19}$, Masahiro Kanai ${ }^{5,19,374,}$

Kristin Tsuo ${ }^{5,19,375}$, Nikolas Baya ${ }^{5,19}$, Patrick Turley ${ }^{5,19}$, Rahul Gupta ${ }^{5,19}$, Raymond K. Walters ${ }^{5,19}$, Duncan S. Palmer ${ }^{5,19}$, Gopal Sarma, ${ }^{5,19}$, Matthew Solomonson ${ }^{5,19}$, Nathan Cheng ${ }^{5,19}$, Wenhan $\mathrm{Lu}^{5,19}$, Claire Churchhouse ${ }^{5,19}$, Jacqueline I. Goldstein ${ }^{5,19}$, Daniel King ${ }^{5,19}$,

Wei Zhou, ${ }^{5,19}$, Cotton Seed ${ }^{5,19}$, Mark J. Daly ${ }^{2,3,5}$, Benjamin M. Neale ${ }^{5,19}$, Hilary Finucane ${ }^{5,19}$, Sam Bryant ${ }^{3}$, F. Kyle Satterstrom ${ }^{5,19}$, Gavin Band ${ }^{376}$, Sarah G Earle ${ }^{369}$, Shang-Kuan $\operatorname{Lin}^{369}$, Nicolas Arning ${ }^{369}$, Nils Koelling ${ }^{370}$

\section{Data Collection Member}

Jacob Armstrong ${ }^{369}$,

Justine K Rudkin ${ }^{369}$

\section{Admin Team Member}

Shawneequa Callier ${ }^{377}$, Sam Bryant ${ }^{5,19}$, Caroline Cusick ${ }^{19}$

\section{UK Blood Donors Cohort}

\section{Analysis Team Lead}


Nicole Soranzo ${ }^{378,379,380}$, Jing Hua Zhao ${ }^{381}$

\section{Data Collection Lead}

John Danesh ${ }^{381,382,383,384,385}$

Emanuele Di Angelantonio ${ }^{381,382,383,384}$

Analysis Team Member

Adam S. Butterworth ${ }^{381,382,383,384}$

\section{VA Million Veteran Program (MVP)}

Analysis Team Lead

Yan V Sun ${ }^{386,387}$,

Jennifer E Huffman ${ }^{388}$

Data Collection Lead

Kelly Cho ${ }^{389}$

\section{Admin Team Lead}

Christopher J O'Donnell ${ }^{388}$,

Phil Tsao ${ }^{390,391}$,

J. Michael Gaziano 389

\section{Analysis Team Member}

Gina Peloso ${ }^{388,392}$

Data Collection Member

Yuk-Lam Ho ${ }^{389}$

\section{Vanda COVID}

Analysis Team Lead

Sandra P Smieszek ${ }^{393}$

\section{Admin Team Lead}

Christos Polymeropoulos ${ }^{393}$, Vasilios Polymeropoulos ${ }^{393}$, Mihael H Polymeropoulos ${ }^{393}$

Analysis Team Member

Bartlomiej P Przychodzen ${ }^{393}$

\section{Variability in immune response genes and severity of SARS-CoV-2 infection (INMUNGEN-CoV2 study)}


medRxiv preprint doi: https://doi.org/10.1101/2021.11.08.21265944; this version posted February 9, 2022. The copyright holder for this preprint (which was not certified by peer review) is the author/funder, who has granted medRxiv a license to display the preprint in perpetuity.

It is made available under a CC-BY-NC-ND 4.0 International license.

Analysis Team Lead

Israel Fernandez-Cadenas ${ }^{394}$

Data Collection Lead

Anna M Planas ${ }^{395,396}$

Analysis Team Member

Jordi Perez-Tur ${ }^{397,398,399}$,

Laia Llucià-Carol ${ }^{394,400}$,

Natalia Cullell ${ }^{394,401}$,

Elena Muiño ${ }^{394}$,

Jara Cárcel-Márquez ${ }^{394}$,

Marta L DeDiego ${ }^{402}$,

Lara Lloret Iglesias ${ }^{403}$

\section{Data Collection Member}




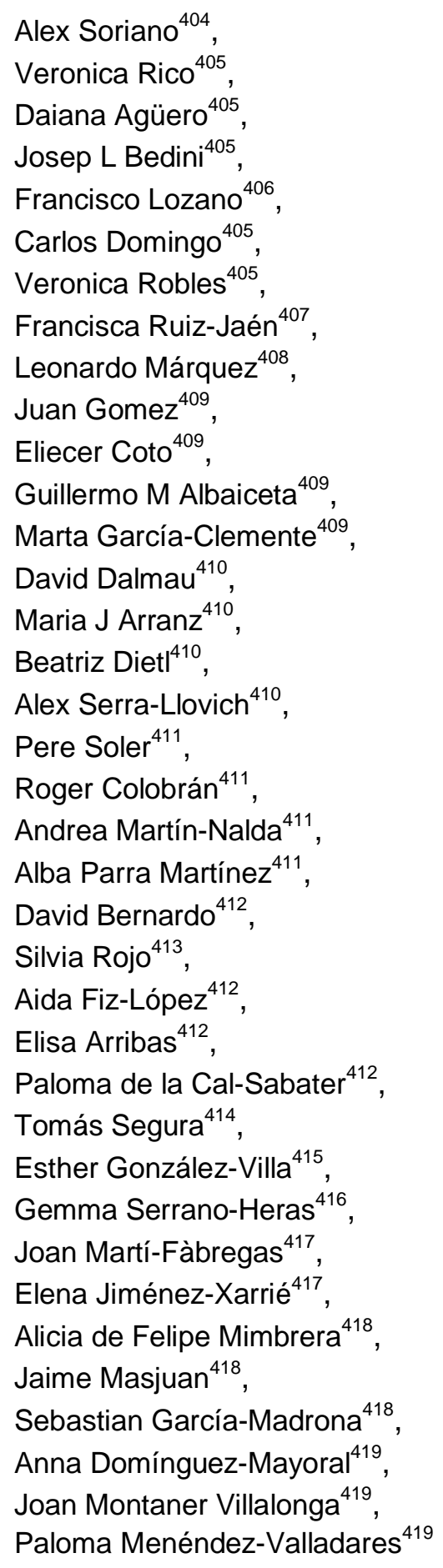

\section{Women's Genome Health Study (WGHS), i.e. the genomics subset of Women's Health Study (WHS)}

\section{Analysis Team Lead}


Daniel I. Chasman ${ }^{420,421}$

Data Collection Lead

Howard D. Sesso ${ }^{420,421}$,

JoAnn E. Manson ${ }^{420,421}$

Admin Team Lead

Julie E. Buring ${ }^{420,421}$,

Paul M Ridker ${ }^{420,421}$

\section{Analysis Team Member}

Giulianini Franco ${ }^{420}$

\section{COVID-19 HGI corresponding authors}

\section{COVID-19 HGI corresponding authors}

Benjamin M Neale ${ }^{4}$, Mark Daly ${ }^{2,3,5}$,

Andrea Ganna ${ }^{2,3,5}$

1. Yale University, New Haven, CT, USA

2. Institute for Molecular Medicine Finland (FIMM), Univerisity of Helsinki, Helsinki, Finland

3. Broad Institute of MIT and Harvard, Cambridge, MA, USA

4. Massachusetts General Hospital, Broad Institute of MIT and Harvard, Cambridge, MA, USA

5. Analytic and Translational Genetics Unit, Massachusetts General Hospital, Boston, MA, USA

6. Icahn School of Medicine at Mount Sinai, New York, NY, USA

7. Institute for Molecular Medicine Finland (FIMM), University of Helsinki, Helsinki, Finland

8. Icahn School of Medicine at Mount Sinai, Genetics and Genomic Sciences

9. Centre for Bioinformatics and Data Analysis, Medical University of Bialystok, Bialystok, Poland

10. University of Michigan, Ann Arbor, MI, USA

11. Ancestry, Lehi, UT, USA

12. Institute for Molecular Medicine Finland (FIMM), Helsinki, Finland

13. University of Liege, GIGA-Institute, Liège, Belgium

14. CHC Mont-Légia, Liège, Belgium

15. 5BHUL (Liège Biobank), CHU of Liège, Liège, Belgium

16. Institute for Molecular Medicine Finland, University of Helsinki, Helsinki, Finland

17. Analytic \& Translational Genetics Unit, Massachusetts General Hospital, Boston, MA, USA

18. Stanley Center for Psychiatric Research, Broad Institute of MIT and Harvard, Cambridge, MA, USA

19. Program in Medical and Population Genetics, Broad Institute of MIT and Harvard, Cambridge, MA, USA

20. CHU of Liege, Liège, Belgium

21. University of Liege, Liège, Belgium

22. $\mathrm{CHU}$ of liege, Liège, Belgium

23. Department of Human Genetics, McGill University, Montréal, Québec, Canada

24. Lady Davis Institute, Jewish General Hospital, McGill University, Montréal, Québec, Canada 
medRxiv preprint doi: https://doi.org/10.1101/2021.11.08.21265944; this version posted February 9, 2022. The copyright holder for this preprint (which was not certified by peer review) is the author/funder, who has granted medRxiv a license to display the preprint in perpetuity.

It is made available under a CC-BY-NC-ND 4.0 International license .

25. Kyoto-McGill International Collaborative School in Genomic Medicine, Graduate School of Medicine, Kyoto University, Kyoto, Japan

26. Research Fellow, Japan Society for the Promotion of Science

27. McGill Genome Centre and Department of Human Genetics, McGill University, Montréal, Québec, Canada

28. Department of Human Genetics, Epidemiology, Biostatistics and Occupational Health, McGill

University, Montréal, Québec, Canada

29. Department of Twin Research, King's College London, London, United Kingdom

30. Department of Epidemiology, Biostatistics and Occupational Health, McGill University, Montréal,

Québec, Canada

31. Department of Emergency Medicine, McGill University, Montréal, Québec, Canada

32. Emergency Department, Jewish General Hospital, McGill University, Montréal, Québec, Canada

33. McGill AIDS Centre, Department of Microbiology and Immunology, Lady Davis Institute for Medical

Research, Jewish General Hospital, McGill University, Montréal, Québec, Canada

34. McGill Centre for Viral Diseases, Lady Davis Institute, Department of Infectious Disease, Jewish

General Hospital, Montréal, Québec, Canada

35. Research Centre of the Centre Hospitalier de l'Université de Montréal, Montréal, Canada

36. Department of Medicine,Research Centre of the Centre Hospitalier de l'Université de Montréal, Montréal, Canada

37. Department of Medicine, Université de Montréal, Montréal, Canada

38. Department of Medicine and Human Genetics, McGill University, Montréal, Québec, Canada

39. Department of Intensive Care, Research Centre of the Centre Hospitalier de l'Université de Montréal, Montréal, Canada

40. Division of Infectious Diseases, Research Centre of the Centre Hospitalier de l'Université de Montréal, Montréal, Canada

41. MRC Human Genetics Unit, Institute of Genetics and Cancer, University of Edinburgh, Western

General Hospital, Edinburgh, UK EH4 2XU

42. Center for Applied Genomics, Children's Hospital of Philadelphia, Philadelphia, PA, USA

43. Department of Pediatrics, Perelman School of Medicine, University of Pennsylvania, Philadelphia, PA, USA

44. Vanderbilt University Medical Center

45. Centre for Genomic and Experimental Medicine, Institute of Genetics and Cancer, University of

Edinburgh, Western General Hospital, Edinburgh, UK, EH4 2XU, Usher Institute, University of Edinburgh, Nine, Edinburgh Bioquarter, 9 Little France Road, Edinburgh, UK, EH16 4UX

46. University of Texas Health

47. Centre for Genomic and Experimental Medicine, Institute of Genetics and Cancer, University of Edinburgh, Western General Hospital, Edinburgh, UK, EH4 2XU

48. Department of Psychology, University of Edinburgh, Edinburgh, UK, EH8 9JZ, Centre for Genomic and Experimental Medicine, Institute of Genetics and Cancer, University of Edinburgh, Western General Hospital, Edinburgh, UK, EH4 2XU

49. University of North Carolina at Chapel Hill

50. Center for Applied Genomics, The Children's Hospital of Philadelphia, Philadelphia, PA, USA

51. Center for Applied Genomics, The Children's Hospital of Philadelphia, Philadelphia, PA, USA

52. Division of Human Genetics, Department of Pediatrics, The Perelman School of Medicine, University of Pennsylvania, Philadelphia, PA, USA

53. Divisions of Human Genetics and Pulmonary Medicine, Department of Pediatrics, The Perelman

School of Medicine, University of Pennsylvania, Philadelphia, PA, USA

54. Faculty of Medicine, University of Iceland, Reykjavik, Iceland

55. University of Colorado - Anschutz Medical Campus, Aurora, CO, USA 
medRxiv preprint doi: https://doi.org/10.1101/2021.11.08.21265944; this version posted February 9, 2022. The copyright holder for this preprint (which was not certified by peer review) is the author/funder, who has granted medRxiv a license to display the preprint in perpetuity.

It is made available under a CC-BY-NC-ND 4.0 International license .

56. MRC Human Genetics Unit, Institute of Genetics and Cancer, University of Edinburgh, Western General Hospital, Edinburgh, Scotland

57. Centre for Global Health Research, Usher Institute, University of Edinburgh, Teviot Place, Edinburgh, Scotland

58. The Roslin Institute, The Royal (Dick) School of Veterinary Studies, University of Edinburgh, Edinburgh, UK

59. Department of Clinical Pathology, Faculty of Medicine, Mansoura University, Mansoura, Egypt.

60. Department of Biosciences, School of Science and Technology, Nottingham Trent University, Nottingham, UK

61. Genolier Innovation Network and Hub, Swiss Medical Network, Genolier Healthcare Campus, Route du Muids 3, 1272 Genolier, Switzerland.

62. Department of Pediatrics, Faculty of Medicine, Mansoura University, Mansoura, Egypt.

63. Department of Clinical Pathology, Faculty of Medicine, Tanta University, Tanta, Egypt.

64. Stanley Center for Psychiatric Research \& Program in Medical and Population Genetics, Broad Institute of MIT and Harvard, Cambridge, MA, USA

65. Chest Department, Faculty of Medicine, Mansoura University, Mansoura, Egypt.

66. Anesthesia, Surgical Intensive Care \& Pain Management Department, Faculty of Medicine, Tanta University, Tanta, Egypt.

67. Department of Medical Biochemistry, Faculty of Medicine, Mansoura University, Mansoura, Egypt.

68. Department of Surgery, Faculty of Medicine, Mansoura University, Mansoura, Egypt.

69. Department of Tropical Medicine, Faculty of Medicine, Mansoura University, Mansoura, Egypt.

70. pediatric and neonatology, Kafr Elzayat General Hospital, Kafr El-Zayat, Egypt.

71. Chest Department, Faculty of Medicine, Tanta University, Tanta, Egypt.

72. Pediatrics Department, Faculty of Medicine, Tanta University, Tanta, Egypt.

73. Department of Anaethesia and Critical Care, Faculty of Medicine, Mansoura University, Mansoura, Egypt.

74. Department of Internal Medicine, Faculty of Medicine, Tanta University, Tanta, Egypt.

75. Faculty of Science, Tanta University, Tanta, Egypt.

76. Department of Internal Medicine

77. Department of Epidemiology

78. Estonian Genome Centre, Institute of Genomics, University of Tartu, Tartu, Estonia

79. SYNLAB Estonia, University of Tartu, Tartu, Estonia

80. Kuressaare Hospital, Kuressaare, Estonia

81. University of Tartu, Tartu, Estonia

82. Institute of Biomedicine and Translational Medicine, University of Tartu

83. Institute of Biomedicine and Translational Medicine, University of Tartu, Tartu, Estonia

84. West Tallinn Central Hospital, Tallinn, Estonia

85. University of Tartu, Tartu University Hospital, Tartu, Estonia

86. Estonian Health Insurance Fund, Tallinn, Estonia

87. Tartu University Hospital, Tartu, Estonia

88. Department of Health Sciences, University of Leicester, Leicester, UK

89. Leicester NIHR Biomedical Research Centre, Leicester, UK

90. Department of Respiratory Sciences, University of Leicester, UK

91. Department of Genetics and Genome Biology, University of Leicester

92. FinnGen, Helsinki, Finland

93. Institute for Molecular Medicine Finland (FIMM), HiLIFE, University of Helsinki, Finland

94. Public Health, Faculty of Medicine, University of Helsinki, Finland

95.

96. Finnish Institute for Health and Welfare (THL), Helsinki, Finland 
medRxiv preprint doi: https://doi.org/10.1101/2021.11.08.21265944; this version posted February 9, 2022. The copyright holder for this preprint (which was not certified by peer review) is the author/funder, who has granted medRxiv a license to display the preprint in perpetuity.

It is made available under a CC-BY-NC-ND 4.0 International license .

97. University of Helsinki, Faculty of Medicine, Clinical and Molecular Metabolism Research Program, Helsinki, Finland

98. Institute of Molecular and Clinical Ophthalmology Basel (IOB), Basel, Switzerland

99. Department of Ophthalmology, University of Basel, Basel, Switzerland

100. Infectious Diseases Service, Department of Medicine, University Hospital and University of

Lausanne, Lausanne, Switzerland

101. Infectious Diseases Service, Department of Medicine, University Hospital, University of Lausanne, Lausanne, Switzerland

102. Centre for Primary Care and Public Health, University of Lausanne, Lausanne, Switzerland.

103. Division of Infectious Diseases and Hospital Epidemiology, Cantonal Hospital St Gallen, St Gallen, Switzerland.

104. Division of Intensive Care, Geneva University Hospitals and the University of Geneva Faculty of Medicine, Geneva, Switzerland.

105. Infectious Disease Service, Department of Internal Medicine, Geneva University Hospital, Geneva, Switzerland.

106. Clinique de Médecine et spécialités, Infectiologie, HFR-Fribourg, Fribourg, Switzerland

107. Infectious Diseases Division, University Hospital Centre of the canton of Vaud, hospital of Valais,

Sion, Switzerland

108. Functional Host Genomics of Infectious Diseases, University Hospital and University of Lausanne, Lausanne, Switzerland

109. Registry COVID, University Hospital and University of Lausanne, Lausanne, Switzerland

110. Pneumonia prediction using lung ultrasound, University Hospital and University of Lausanne,

Lausanne, Switzerland

111. Center for Primary Care and Public Health (Unisanté), University of Lausanne, Lausanne,

Switzerland

112. Covid-19 Risk Prediction in Swiss ICUs-Trial, Division of Infectious Diseases and Hospital

Epidemiology, Cantonal Hospital St Gallen, St Gallen, Switzerland.

113. GCAT-Genomes for Life, Germans Trias i Pujol Health Sciences Research Institute (IGTP), Crta. de Can Ruti, Cami de les Escoles s/n.08916 Badalona, Catalonia

114. Catalan Institute of Oncology,Bellvitge Biomedical Research Institute, Consortium for Biomedical

Research in Epidemiology and Public Health and University of Barcelona, Barcelona, Spain

115. ISGlobal, Barcelona, Spain

116. IMIM (Hospital del Mar Medical Research Institute), Barcelona, Spain

117. Universitat Pompeu Fabra (UPF), Barcelona, Spain

118. CIBER Epidemiología y Salud Pública (CIBERESP), Madrid, Spain

119. Barcelona Supercomputing Center - Centro Nacional de Supercomputación (BSC-CNS).Life \& Medical Sciences *currently at Programs in Metabolism and Medical and Population Genetics, Broad Institute of MIT and Harvard, Cambridge, MA, USA and Diabetes Unit and Center for Genomic Medicine, Massachusetts General Hospital, Boston, MA, USA. Harvard Medical School, Boston, Massachusetts, USA

120. Barcelona Supercomputing Center - Centro Nacional de Supercomputación (BSC-CNS).Life \& Medical Sciences, Barcelona, Spain

121. University of Siena, DIISM-SAILAB, Siena, Italy

122. Université Côte d'Azur, Inria, CNRS, I3S, Maasai, Nice, France

123. Medical Genetics, University of Siena, Italy

124. Genetica Medica, Azienda Ospedaliero-Universitaria Senese, Italy

125. Med Biotech Hub and Competence Center, Department of Medical Biotechnologies, University of Siena, Siena, Italy 
medRxiv preprint doi: https://doi.org/10.1101/2021.11.08.21265944; this version posted February 9, 2022. The copyright holder for this preprint (which was not certified by peer review) is the author/funder, who has granted medRxiv a license to display the preprint in perpetuity.

It is made available under a CC-BY-NC-ND 4.0 International license .

126. Division of Infectious Diseases and Immunology, Department of Medical Sciences and Infectious Diseases, Fondazione IRCCS Policlinico San Matteo, Pavia, Italy

127. Department of Internal Medicine and Therapeutics, University of Pavia, Italy

128. Department of Infectious and Tropical Diseases, University of Brescia and ASST Spedali Civili Hospital, Brescia, Italy

129. Chirurgia Vascolare, Ospedale Maggiore di Crema, Crema, Italy

130. III Infectious Diseases Unit, ASST-FBF-Sacco, Milan, Italy

131. Department of Biomedical and Clinical Sciences Luigi Sacco, University of Milan, Milan, Italy

132. Med Biotech Hub and Competence Center, Department of Medical Biotechnologies, University of Siena, Italy

133. Dept of Specialized and Internal Medicine, Tropical and Infectious Diseases Unit, Azienda Ospedaliera Universitaria Senese, Siena, Italy

134. Unit of Respiratory Diseases and Lung Transplantation, Department of Internal and Specialist Medicine, University of Siena, Siena, Italy

135. Dept of Emergency and Urgency, Medicine, Surgery and Neurosciences, Unit of Intensive Care Medicine, Siena University Hospital, Siena, Italy

136. Department of Medical, Surgical and Neuro Sciences and Radiological Sciences, Unit of Diagnostic Imaging, University of Siena, Siena, Italy

137. Rheumatology Unit, Department of Medicine, Surgery and Neurosciences, University of Siena, Policlinico Le Scotte, Siena, Italy

138. Department of Specialized and Internal Medicine, Infectious Diseases Unit, San Donato Hospital Arezzo, Arezzo, Italy

139. Dept of Emergency, Anesthesia Unit, San Donato Hospital, Arezzo, Italy

140. Department of Specialized and Internal Medicine, Pneumology Unit and UTIP, San Donato Hospital, Arezzo, Italy

141. Department of Emergency, Anesthesia Unit, Misericordia Hospital, Grosseto, Italy

142. Department of Specialized and Internal Medicine, Infectious Diseases Unit, Misericordia Hospital, Grosseto, Italy

143. Department of Preventive Medicine, Azienda USL Toscana Sud Est, Arezzo, Italy

144. Clinical Chemical Analysis Laboratory, Misericordia Hospital, Grosseto, Italy

145. Territorial Scientific Technician Department, Azienda USL Toscana Sud Est, Arezzo, Italy

146. Clinical Chemical Analysis Laboratory, San Donato Hospital, Arezzo, Italy

147. Department of Health Sciences, Clinic of Infectious Diseases, ASST Santi Paolo e Carlo, University of Milan, Milan, Italy

148. Department of Anesthesia and Intensive Care, University of Modena and Reggio Emilia, Modena, Italy

149. HIV/AIDS Department, National Institute for Infectious Diseases, IRCCS, Lazzaro Spallanzani, Rome, Italy

150. Infectious Diseases Clinic, Department of Medicine 2, Azienda Ospedaliera di Perugia and

University of Perugia, Santa Maria Hospital, Perugia, Italy

151. Infectious Diseases Clinic, "Santa Maria" Hospital, University of Perugia, Perugia, Italy

152. Department of Infectious Diseases, Treviso Hospital, Treviso, Italy

153. Clinical Infectious Diseases, Mestre Hospital, Venezia, Italy

154. Infectious Diseases Clinic, ULSS1, Belluno, Italy

155. Medical Genetics and Laboratory of Medical Genetics Unit, A.O.R.N. "Antonio Cardarelli", Naples, Italy

156. Department of Molecular Medicine and Medical Biotechnology, University of Naples Federico II, Naples, Italy

157. CEINGE Biotecnologie Avanzate, Naples, Italy 
medRxiv preprint doi: https://doi.org/10.1101/2021.11.08.21265944; this version posted February 9, 2022. The copyright holder for this preprint (which was not certified by peer review) is the author/funder, who has granted medRxiv a license to display the preprint in perpetuity.

It is made available under a CC-BY-NC-ND 4.0 International license .

158. IRCCS SDN, Naples, Italy

159. Unit of Respiratory Physiopathology, AORN dei Colli, Monaldi Hospital, Naples, Italy

160. Division of Medical Genetics, Fondazione IRCCS Casa Sollievo della Sofferenza Hospital, San

Giovanni Rotondo, Italy

161. Department of Medical Sciences, Fondazione IRCCS Casa Sollievo della Sofferenza Hospital, San

Giovanni Rotondo, Italy

162. Infectious Diseases Clinic, Policlinico San Martino Hospital, IRCCS for Cancer Research, Genova, Italy

163. Microbiology, Fondazione Policlinico Universitario Agostino Gemelli IRCCS, Catholic University of Medicine, Rome, Italy

164. Department of Laboratory Sciences and Infectious Diseases, Fondazione Policlinico Universitario A. Gemelli IRCCS, Rome, Italy

165. Department of Cardiovascular Diseases, University of Siena, Siena, Italy

166. Otolaryngology Unit, University of Siena, Siena, Italy

167. Department of Internal Medicine, ASST Valtellina e Alto Lario, Sondrio, Italy

168. First Aid Department, Luigi Curto Hospital, Polla, Salerno, Italy

169. U.O.C. Laboratorio di Genetica Umana, IRCCS Istituto G. Gaslini, Genova, Italy

170. Infectious Diseases Clinics, University of Modena and Reggio Emilia, Modena, Italy

171. U.O.C. Medicina, ASST Nord Milano, Ospedale Bassini, Cinisello Balsamo (MI), Italy

172. Department of Cardiovascular, Neural and Metabolic Sciences, Istituto Auxologico Italiano, IRCCS, San Luca Hospital, Milan, Italy

173. Department of Medicine and Surgery, University of Milano-Bicocca, Milan, Italy

174. Istituto Auxologico Italiano, IRCCS, Center for Cardiac Arrhythmias of Genetic Origin, Milan, Italy

175. Istituto Auxologico Italiano, IRCCS, Laboratory of Cardiovascular Genetics, Milan, Italy

176. Unit of Infectious Diseases, ASST Papa Giovanni XXIII Hospital, Bergamo, Italy

177. Department of Cardiology, Istituti Clinici Scientifici Maugeri IRCCS, Institute of Montescano, Pavia, Italy

178. Istituti Clinici Scientifici Maugeri, IRCCS, Department of Cardiac Rehabilitation, Institute of Tradate (VA), Pavia, Italy

179. Cardiac Rehabilitation Unit, Fondazione Salvatore Maugeri, IRCCS, Scientific Institute of Milan, Milan, Italy

180. IRCCS C. Mondino Foundation, Pavia, Italy

181. Medical Genetics Unit, Meyer Children's University Hospital, Florence, Italy

182. Department of Medicine, Pneumology Unit, Misericordia Hospital, Grosseto, Italy

183. Department of Preventive Medicine, Azienda USL Toscana Sud Est, Tuscany, Italy

184. Department of Anesthesia and Intensive Care Unit, ASST Fatebenefratelli Sacco, Luigi Sacco

Hospital, Polo Universitario, University of Milan, Milan, Italy

185. Infectious Disease Unit, Hospital of Massa, Massa, Italy.

186. Department of Clinical Medicine, Public Health, Life and Environment Sciences, University of

L'Aquila, L'Aquila, Italy

187. UOSD Laboratorio di Genetica Medica - ASL Viterbo, San Lorenzo, Italy

188. Department of Medical Sciences, Infectious and Tropical Diseases Unit, Azienda Ospedaliera

Universitaria Senese, Siena, Italy

189. Unit of Infectious Diseases, S.M. Annunziata Hospital, Florence, Italy.

190. IRCCS Mondino Foundation, Pavia, Italy.

191. Infectious Disease Unit, Hospital of Lucca, Lucca, Italy

192. Health Management, Azienda USL Toscana Sudest, Tuscany, Italy

193. Department of Mathematics, University of Pavia, Pavia, Italy

194. Independent Researcher, Milan, Italy 
medRxiv preprint doi: https://doi.org/10.1101/2021.11.08.21265944; this version posted February 9, 2022. The copyright holder for this preprint (which was not certified by peer review) is the author/funder, who has granted medRxiv a license to display the preprint in perpetuity.

It is made available under a CC-BY-NC-ND 4.0 International license .

195. Department of Electronics, Information and Bioengineering (DEIB), Politecnico di Milano, Milano, Italy

196. Scuola Normale Superiore, Pisa, Italy

197. CNR-Consiglio Nazionale delle Ricerche, Istituto di Biologia e Biotecnologia Agraria (IBBA), Milano, Italy

198. CNR-Consiglio Nazionale delle Ricerche, Istituto di Biologia e Biotecnologia Agraria (IBBA), Milano, Italy

199. Med Biotech Hub and Competence Center, Department of Medical Biotechnologies, University of

Siena, Siena, Italy Italy

200. Core Research Laboratory, ISPRO, Florence, Italy

201. Genetica Medica, Azienda Ospedaliero-Universitaria Senese, Siena, Italy

202. Infectious Diseases Clinic, Department of Medicine 2, Azienda Ospedaliera di Perugia and

University of Perugia, Santa Maria Hospital

203. Division of Infectious Diseases and Immunology, Fondazione IRCCS Policlinico San Matteo, Pavia, Italy

204. Fondazione IRCCS Ca' Granda Ospedale Maggiore Policlinico

205. Department of Molecular and Translational Medicine, University of Brescia, Italy

206. Clinical Chemistry Laboratory, Cytogenetics and Molecular Genetics Section, Diagnostic

Department, ASST Spedali Civili di Brescia, Brescia, Italy

207. Department of Medical and Surgical Sciences for Children and Adults, University of Modena and

Reggio Emilia, Modena, Italy

208. Department of Molecular Medicine, University of Padova, Padua, Italy

209. Laboratory of Regulatory and Functional Genomics, Fondazione IRCCS Casa Sollievo della

Sofferenza, San Giovanni Rotondo (Foggia), Italy

210. Clinical Trial Office, Fondazione IRCCS Casa Sollievo della Sofferenza Hospital, San Giovanni

Rotondo, Italy

211. Department of Health Sciences, University of Genova, Genova, Italy

212. Oncologia Medica e Ufficio Flussi Sondrio, Sondrio, Italy

213. Local Health Unit-Pharmaceutical Department of Grosseto, Toscana Sud Est Local Health Unit,

Grosseto, Italy

214. Department of Respiratory Diseases, Azienda Ospedaliera di Cremona, Cremona, Italy

215. Direzione Scientifica, Istituti Clinici Scientifici Maugeri IRCCS, Pavia, Italy

216. Fondazione per la ricerca Ospedale di Bergamo, Bergamo, Italy

217. Department of Cardiovascular, Neural and Metabolic Sciences, Istituto Auxologico Italiano, IRCCS,

San Luca Hospital, Milan, Italy

218. Allelica Inc, New York, NY, USA

219. Dept of Specialized and Internal Medicine, Tropical and Infectious Diseases Unit, Azienda

Ospedaliera Universitaria Senese, Siena, Italy

220. Department of Clinical and Experimental Medicine, Infectious Diseases Unit, University of Pisa, Pisa, Italy.

221. Infectious Disease Unit, Santo Stefano Hospital, AUSL Toscana Centro, Prato, Italy.

222. Clinic of Infectious Diseases, Catholic University of the Sacred Heart, Rome, Italy.

223. Medical Genetics, University of Siena, Siena, Italy

224. Infectious Disease Unit, Hospital of Lucca, Lucca Italy.

225. Department of Diagnostic and Laboratory Medicine, Institute of Biochemistry and Clinical

Biochemistry, Fondazione Policlinico Universitario A. Gemelli IRCCS, Catholic University of the Sacred

Heart, Rome, Italy.

226. MRC Human Genetics Unit, IGC,University of Edinburgh.EH4 2XU,UK 
medRxiv preprint doi: https://doi.org/10.1101/2021.11.08.21265944; this version posted February 9, 2022. The copyright holder for this preprint (which was not certified by peer review) is the author/funder, who has granted medRxiv a license to display the preprint in perpetuity.

It is made available under a CC-BY-NC-ND 4.0 International license .

227. Medical Genetics Section, Centre for Genomic and Experimental Medicine, IGC, University of Edinburgh, Edinburgh, UK, Generation Scotland, Centre for Genomic and Experimental Medicine, IGC, University of Edinburgh, Edinburgh, UK

228. Department of Psychology, University of Edinburgh, Edinburgh, UK, Medical Genetics Section, Centre for Genomic and Experimental Medicine, IGC, University of Edinburgh, Edinburgh, UK, Generation Scotland, Centre for Genomic and Experimental Medicine, IGC, University of Edinburgh, Edinburgh, UK 229. Blizard Institute, Queen Mary University of London, London, United Kingdom 230. School of Basic and Medical Biosciences, Faculty of Life Sciences and Medicine, King's College London, London, United Kingdom

231. Medical and Population Genomics, Wellcome Sanger Institute, Hinxton, UK

232. Bradford Institute for Health Research, Bradford Teaching Hospitals National Health Service (NHS)

Foundation Trust, Bradford, UK

233. Blizard Institute, 4 Newark Street, Queen Mary University of London

234. Institute of Population Health Sciences, 4 Newark Street, Queen Mary University of London, London, United Kingdom

235. Genes \& Health, Blizard Institute, Queen Mary University of London, E1 2AT, London, United Kingdom

236. Institute of Population Health Sciences, 4 Newark Street, Queen Mary University of London

237. Department of Biostatistics, University of Michigan, Ann Arbor, MI, USA

238. Digestive Oncology Research Center, Digestive Disease Research Institute, Shariati Hospital, Tehran University of Medical Sciences, Tehran, Iran

239. Department of Pulmonology, School of Medicine, Shariati Hospital, Tehran University of Medical Sciences, Tehran, Iran

240. Department of Critical Care Medicine, Noorafshar Hospital, Tehran, Iran

241. Department of Emergency Intensive Care Unit, School of Medicine, Shariati Hospital, Tehran University of Medical Sciences, Tehran, Iran

242. Department of Anesthesiology, School of Medicine, Amir Alam Hospital, Tehran University of Medical Sciences, Tehran, Iran

243. Department of Pulmonology, School of Medicine, Tehran University of Medical Sciences, Tehran, Iran

244. Department of Pathology, Parseh Pathobiology and Genetics Laboratory, Tehran, Iran

245. Department of Microbiology, Health and Family Research Center, NIOC Hospital, Tehran, Iran 246. Department of Emergency Medicine, School of Medicine, Shariati Hospital, Tehran University of Medical Sciences, Tehran, Iran

247. Department of Anesthesiology, School of Medicine, Tehran University of Medical Sciences, Tehran, Iran

248. Department of Pathology, Faculty of Medicine, Tehran Azad University, Tehran, Iran

249. Clinical Pharmacokinetics and Pharmacogenomics Research Unit, Faculty of Medicine, Chulalongkorn University, Bangkok Thailand

250. Department of Pharmacology, Faculty of Medicine, Chulalongkorn University, Bangkok Thailand 251. Thai Red Cross Emerging Infectious Diseases Clinical Centre, King Chulalongkorn Memorial Hospital, Bangkok, Thailand

252. Department of Pediatrics, Faculty of Medicine, Chulalongkorn University, Bangkok, Thailand.

253. Immunology Division, Department of Microbiology, Faculty of Medicine, Chulalongkorn University, Bangkok, Thailand

254. Center of Excellence in Immunology and Immune-mediated Diseases, Department of Microbiology, Faculty of Medicine, Chulalongkorn University, Bangkok, Thailand. 
medRxiv preprint doi: https://doi.org/10.1101/2021.11.08.21265944; this version posted February 9, 2022. The copyright holder for this preprint (which was not certified by peer review) is the author/funder, who has granted medRxiv a license to display the preprint in perpetuity.

It is made available under a CC-BY-NC-ND 4.0 International license .

255. Clinical Pharmacokinetics and Pharmacogenomics Research Unit, Faculty of Medicine, Chulalongkorn University, Bangkok, Thailand

256. Department of Pathology, Faculty of Medicine, Nakornnayok, Srinakharinwirot University, Thailand 257. Center of Excellence for Medical Genomics, Medical Genomics Cluster, Department of Pediatrics, Faculty of Medicine, Chulalongkorn University, Bangkok, Thailand.

258. Excellence Center for Genomics and Precision Medicine, King Chulalongkorn Memorial Hospital, The Thai Red Cross Society, Bangkok, Thailand.

259. Department of Mathematics and Computer Science, Faculty of Science, Chulalongkorn University, Bangkok, Thailand

260. Omics Sciences and Bioinfomatics Center, Faculty of Science, Chulalongkorn University, Bangkok, Thailand

261. Research Affairs, Faculty of Medicine, Chulalongkorn University, Bangkok, Thailand

262. Center of Excellence for Medical Genomics, Medical Genomics Cluster, Faculty of Medicine, Chulalongkorn University, Bangkok, Thailand

263. Division of Infectious Disease, Department of Medicine, Faculty of Medicine, Chulalongkorn University, Bangkok, Thailand.

264. Center of Excellence in Pediatric Infectious Diseases and Vaccines, Chulalongkorn University, Bangkok, Thailand

265. Department of Microbiology, Faculty of Medicine, Chulalongkorn University, Bangkok, Thailand

266. Division of Infectious Diseases, Department of Medicine, Faculty of Medicine, Chulalongkorn University, Bangkok, Thailand

267. Healthcare-associated Infection Research Group STAR (Special Task Force for Activating Research), Chulalongkorn University, Bangkok, Thailand

268. K.G. Jebsen Center for Genetic Epidemiology, Department of Public Health and Nursing, NTNU, Norwegian University of Science and Technology, Trondheim, 7030, Norway

269. HUNT Research Center, Department of Public Health and Nursing, NTNU, Norwegian University of Science and Technology, Levanger, 7600, Norway

270. Clinic of Medicine, St. Olavs Hospital, Trondheim University Hospital, Trondheim, 7030, Norway

271. Division of Cardiovascular Medicine, Department of Internal Medicine, University of Michigan, Ann

Arbor, MI, USA

272. Department of Computational Medicine and Bioinformatics, University of Michigan, Ann Arbor, MI, USA

273. Department of Human Genetics, University of Michigan, Ann Arbor, MI, USA

274. Analytic and Translational Genetics Unit, Massachusetts General Hospital, Boston, Massachusetts, USA

275. Program in Medical and Population Genetics, Broad Institute of Harvard and MIT, Cambridge, Massachusetts, USA

276. Gemini Center for Sepsis Research, Department of Circulation and Medical Imaging, NTNU, Norwegian University of Science and Technology, Trondheim, Norway

277. Department of Chronic Disease Epidemiology and Center for Perinatal, Pediatric and Environmental Epidemiology, Yale School of Public Health, New Haven, CT, USA

278. Clinic of Anaesthesia and Intensive Care, St. Olavs Hospital, Trondheim University Hospital,

Trondheim, Norway

279. Department of Genetics, University Medical Centre Groningen, University of Groningen, Groningen, Netherlands

280. Department of Epidemiology, University Medical Centre Groningen, University of Groningen, Groningen, Netherlands

281. Department of Genetics, University Medical Centre Groningen, University of Groningen / Department of Genetics, University Medical Centre Utrecht, P.O. Box 85500, 3508 GA, Utrecht, The Netherlands 
medRxiv preprint doi: https://doi.org/10.1101/2021.11.08.21265944; this version posted February 9, 2022. The copyright holder for this preprint (which was not certified by peer review) is the author/funder, who has granted medRxiv a license to display the preprint in perpetuity.

It is made available under a CC-BY-NC-ND 4.0 International license .

282. Department of Epidemiology, University of Groningen, University Medical Center Groningen, Groningen, The Netherlands

283. University of Groningen, University Medical Center Groningen, Department of Genetics, Groningen, The Netherlands

284. Department of Genetics, University Medical Center Groningen, Groningen, The Netherlands

285. Department of Psychiatry, University Medical Center Groningen, Groningen, The Netherlands

286. Center for Genomic Medicine, Massachusetts General Hospital, Boston, MA, USA

287. Programs in Metabolism and Medical and Population Genetics, Broad Institute of MIT and Harvard, Cambridge, MA, USA

288. Diabetes Unit and Center for Genomic Medicine, Massachusetts General Hospital, Boston, MA,

USA. Harvard Medical School, Boston, Massachusetts, USA

289. Channing Division of Network Medicine, Department of Medicine, Brigham and Women's Hospital,

Boston, MA, USA

290. Brigham and Women's Hospital, Boston, MA, USA

291. Psychiatric and Neurodevelopmental Genetics Unit, Center for Genomic Medicine, Massachusetts

General Hospital, Boston, MA

292. Department of Neurology, Massachusetts General Hospital, Boston, MA, USA

293. Division of General Internal Medicine, Massachusetts General Hospital and Dpeartment of Medicine, Harvard Medical School and Program in Medical and Population Genetics, Broad Institute, Boston, MA, USA

294. Division of Genetics, Department of Medicine, Brigham and Women's Hospital, Broad Institute of MIT and Harvard, Harvard Medical School, Boston, MA, USA

295. Division of Genetics, Department of Medicine, Brigham and Women's Hospital, Boston, MA, USA

296. Seaver Autism Center for Research and Treatment

297. Department of Psychiatry

298. Icahn School of Medicine at Mount Sinai, New York, NY 10029, USA

299. Mount Sinai Clinical Intelligence Center, Department of Genetics and Genomic Sciences, Icahn

School of Medicine at Mount Sinai, New York, NY, 10029, USA

300. Department of Genetics and Genomic Sciences, Icahn School of Medicine at Mount Sinai, New

York, NY, 10029, USA

301. Sema4, a Mount Sinai venture, Stamford CT, 06902, USA

302. Seaver Autism Center for Research and Treatment, Department of Psychiatry, Icahn School of Medicine at Mount Sinai, New York, NY 10029, USA

303. Mount Sinai CInical Intelligence Center, Charles Bronfman Institute for Personalized Medicine

304. Department of Genetics \& Genomic Sciences, Icahn School of Medicine at Mount Sinai, New York, NY, 10029, USA

305. Mount Sinai Clinical Intelligence Center, Department of Genetics and Genomic Sciences, Icahn

School of Medicine at Mount Sinai, New York, NY 10029, USA

306. Icahn Institute of Data Science and Genomics Technology, New York, NY 10029, USA

307. Mount Sinai Clinical Intelligence Center, New York, NY 10029, USA

308. Department of Genetics and Genomic Sciences, Icahn School of Medicine at Mount Sinai, New

York, NY, USA

309. Charles Bronfman Institute for Personalized Medicine, Icahn School of Medicine at Mount Sinai, New York, NY, 10029, USA

310. Institute for Genomic Health, Icahn School of Medicine at Mount Sinai, New York, NY, USA

311. The Charles Bronfman Institute for Personalized Medicine, Icahn School of Medicine at Mount Sinai, New York, NY 10029, USA

312. The Mindich Child Health and Development Institute, Icahn School of Medicine at Mount Sinai, New York, NY 10029, USA 
medRxiv preprint doi: https://doi.org/10.1101/2021.11.08.21265944; this version posted February 9, 2022. The copyright holder for this preprint (which was not certified by peer review) is the author/funder, who has granted medRxiv a license to display the preprint in perpetuity.

It is made available under a CC-BY-NC-ND 4.0 International license .

313. Pamela Sklar Division of Psychiatric Genomics, Department of Psychiatry, Department of Genetic and Genomic Sciences

314. Pamela Sklar Division of Psychiatric Genomics, Department of Psychiatry, Department of Genetic and Genomic Sciences, Icahn School of Medicine at Mount Sinai, New York, NY 10029, USA

315. Seaver Autism Center for Research and Treatment, Department of Psychiatry

316. Mount Sinai Clinical Intelligence Center, Department of Psychiatry, Department of Genetic and Genomic Sciences, Icahn School of Medicine at Mount Sinai, NY, 10029, USA

317. Department of Environmental Medicine and Public Health, Icahn School of Medicine at Mount Sinai, New York, NY 10029, USA

318. Mount Sinai Clinical Intelligence Center

319. The Hasso Plattner Institute of Digital Health at Mount Sinai

320. BioMe Phenomics Center, Icahn School of Medicine at Mount Sinai, New York, NY 10029, USA

321. Department of Medicine, Icahn School of Medicine at Mount Sinai, New York, NY 10029, USA

322. Pamela Sklar Division of Psychiatric Genomics, Seaver Autism Center for Research and Treatment, Department of Psychiatry, Department of Genetic and Genomic Sciences, Icahn School of Medicine at Mount Sinai, New York, NY 10029, USA

323. Regeneron Genetics Center, Tarrytown, NY, USA

324. Phenomic Analytics \& Clinical Data Core, Geisinger Health System, Danville, PA, USA

325. Department of Population Health Sciences, Geisinger Health System, Danville, PA, USA

326. Department of Molecular and Functional Genomics, Geisinger Health System, Danville, PA, USA

327. Vrije Universiteit Amsterdam, Amsterdam, UK

328. Department of Genetics, University of Pennsylvania Perelman School of Medicine, Philadelphia, PA, USA

329. Department of Biomedical Data Science, Stanford University, Stanford, CA, USA

330. Genomics Research Department, Saudi Human Genome Project, King Fahad Medical City and King Abdulaziz City for Science and Technology, Riyadh, Saudi Arabia

331. Developmental Medicine Department, King Abdullah International Medical Research Center, King Saud Bin Abdulaziz University for Health Sciences, Ministry of National Guard- Health Affairs, Riyadh, Kingdom of Saudi Arabia and Saudi Human Genome Project (SHGP), King Abdulaziz City for Science and Technology (KACST), Satellite Lab at King Abdulaziz Medical City (KAMC), Ministry of National Guard Health Affairs (MNG-HA), Riyadh , Kingdom of Saudi Arabia.

332. College of Applied Medical Sciences, Taibah University, Madina, Saudi Arabia

333. Developmental Medicine Department, King Abdullah International Medical Research Center, King Saud Bin Abdulaziz University for Health Sciences, Ministry of National Guard Health Affairs, Riyadh, Saudi Arabia

334. Life Science and environmental institute, King Abdulaziz City for Science and Technology, Riyadh, Saudi Arabia

335. The Liver Transplant Unit, King Faisal Specialist Hospital and Research Centre, Riyadh, Saudi Arabia. The Division of Gastroenterology and Hepatology, Johns Hopkins University

336. Department of Pathology, College of Medicine, King Saud University, Riyadh, Saudi Arabia 337. Titus Family Department of Clinical Pharmacy, USC School of Pharmacy University of Southern California 338. KACST-BWH Centre of Excellence for Biomedicine, Joint Centers of Excellence Program, King Abdulaziz City for Science and Technology (KACST), Riyadh, Kingdom of Saudi Arabia 339. Ministry of the National Guard Health Affairs, King Abdullah International Medical Research Center and King Saud Bin Abdulaziz University for Health Sciences, Riyadh, Kingdom of Saudi Arabia 340. Ohud Hospital, Ministry of Health, Madinah,Saudi Arabia 341. Pediatric Infectious Diseases, Children's Specialized Hospital, King Fahad Medical City, Riyadh, Saudi Arabia 
medRxiv preprint doi: https://doi.org/10.1101/2021.11.08.21265944; this version posted February 9, 2022. The copyright holder for this preprint (which was not certified by peer review) is the author/funder, who has granted medRxiv a license to display the preprint in perpetuity. It is made available under a CC-BY-NC-ND 4.0 International license .

342. The Saudi Biobank, King Abdullah International Medical Research Center, King Saud bin Abdulaziz University for Health Sciences, Ministry of National Guard Health Affairs, Riyadh, Saudi Arabia 343. Developmental Medicine Department, King Abdullah International Medical Research Center, King Saud Bin Abdulaziz University for Health Sciences, King AbdulAziz Medical City, Ministry of National Guard Health Affairs, Riyadh, Kingdom of Saudi Arabia

344. Department of Pathology and Laboratory Medicine, King Abdulaziz Medical City, Ministry of National Guard Health Affairs, King Saud Bin Abdulaziz University for Health Sciences and King Abdullah International Medical Research Center, Riyadh, Saudi Arabia

345. Laboratory Department, Security Forces Hospital, General Directorate of Medical Services, Ministry of Interior

346. King Abdulaziz City for Science and Technology (KACST), Riyadh, Kingdom of Saudi Arabia 347. Department of Developmental Medicine, King Abdullah International Medical Research Center, King Saud Bin Abdulaziz University for Health Sciences, King Abdulaziz Medical City, Ministry of National Guard Health Affairs, Riyadh, Saudi Arabia

348. Titus Family Department of Clinical Pharmacy, USC School of Pharmacy

349. Department of Clinical Laboratory Sciences, College of Applied Medical Sciences, King Saud University, Riyadh, Saudi Arabia.

350. Department of Neuroscience, Karolinska Institutet, Stockholm, Sweden

351. Max Planck Institute for Evolutionary Anthropology, Leipzig, Germany

352. Stanley Center for Psychiatric Research \& Program in Medical and Population Genetics

353. Anaesthesiology and Intensive Care Medicine, Department of Surgical Sciences, Uppsala University, Uppsala, Sweden

354. Integrative Physiology, Department of Medical Cell Biology, Uppsala University, Uppsala, Sweden 355. Hedenstierna Laboratory, CIRRUS, Anaesthesiology and Intensive Care Medicine, Department of Surgical Sciences, Uppsala University, Uppsala, Sweden

356. Division Anesthesiology and Intensive Care, CLINTEC, Karolinska Institutet, Stockholm, Sweden

357. Department of Genetics and Bioinformatics, Norwegian Institute of Public Health, Oslo, Norway.

358. Centre for Fertility and Health, Norwegian Institute of Public Health, Oslo, Norway.

359. Department of Method Development and Analytics, Norwegian Institute of Public Health, Oslo, Norway.

360. King's College London, Department of Twin Research and Genetic Epidemiology, London, UK 361. NIHR Biomedical Research Centre at Guy's and St Thomas' Foundation Trust, London, UK

362. Genomics England

363. Queen Mary University, London, United Kingdom

364. William Harvey Research Institute, Barts and the London School of Medicine and Dentistry, Queen Mary University of London, London EC1M 6BQ, UK

365. UCL Great Ormond Street Institute of Child Health, London, United Kingdom

366. Genomics England, London, United Kingdom

367. University of Cambridge, London, United Kingdom

368. Department of Human Genetics, McGill University, Montréal, Québec, Canada. Lady Davis Institute, Jewish General Hospital, McGill University, Montréal, Québec, Canada. Kyoto-McGill International Collaborative School in Genomic Medicine, Graduate School of Medicine, Kyoto University, Kyoto, Japan. Research Fellow, Japan Society for the Promotion of Science

369. Big Data Institute, Nuffield Department of Population Health, University of Oxford, Li Ka Shing Centre for Health Information and Discovery, Old Road Campus, Oxford, OX3 7LF, United Kingdom 370. Genomics PLC, King Charles House, Park End Street, Oxford, OX1 1JD, United Kingdom 371. Nuffield Department of Medicine, Experimental Medicine Division, University of Oxford, John Radcliffe Hospital, Oxford, OX3 9DU, United Kingdom 
medRxiv preprint doi: https://doi.org/10.1101/2021.11.08.21265944; this version posted February 9, 2022. The copyright holder for this preprint (which was not certified by peer review) is the author/funder, who has granted medRxiv a license to display the preprint in perpetuity.

It is made available under a CC-BY-NC-ND 4.0 International license .

372. Public Health England, Field Service, Addenbrooke's Hospital, Cambridge, CB2 0QQ, United Kingdom

373. Public Health England, Data and Analytical Services, National Infection Service, London, NW9 5EQ, United Kingdom

374. Program in Bioinformatics and Integrative Genomics, Harvard Medical School, Boston, MA, USA

375. Program in Biological and Biomedical Sciences, Harvard Medical School, Boston, MA, USA

376. Wellcome Centre for Human Genetics, University of Oxford, Roosevelt Drive, Oxford, OX3 7BN, United Kingdom

377. Department of Clinical Research and Leadership, George Washington University, Washington, DC, USA

378. Department of Human Genetics, The Wellcome Sanger Institute, Wellcome Genome Campus, Hinxton, Cambridge, CB10 1HH, UK

379. The National Institute for Health Research Blood and Transplant Unit in Donor Health and Genomics, University of Cambridge, Strangeways Research Laboratory, Wort's Causeway, Cambridge, CB1 8RN, UK

380. Department of Haematology, University of Cambridge, Cambridge Biomedical Campus, Long Road, Cambridge, CB2 OPT, UK

381. British Heart Foundation Cardiovascular Epidemiology Unit, Department of Public Health and Primary Care, University of Cambridge, Cambridge, UK

382. British Heart Foundation Centre of Research Excellence, University of Cambridge, Cambridge, UK 383. National Institute for Health Research Blood and Transplant Research Unit in Donor Health and Genomics, University of Cambridge, Cambridge, UK

384. Health Data Research UK Cambridge, Wellcome Genome Campus and University of Cambridge, Cambridge, UK

385. Department of Human Genetics, Wellcome Sanger Institute, Hinxton, UK

386. Department of Epidemiology, Emory University Rollins School of Public Health

387. Atlanta CA Health Care System, North Druid Hills, GA, USA

388. Center for Population Genomics, MAVERIC, VA Boston Healthcare System, Boston, MA, USA

389. MAVERIC, VA Boston Healthcare System, Boston, MA, USA

390. Stanford University

391. Palo Alto VA Healthcare System, Stanford, CA, USA

392. Department of Biostatistics, Boston Univeristy School of Public Health

393. Vanda Pharmaceuticals Inc.

394. Stroke Pharmacogenomics and Genetics, Biomedical Research Institute Sant Pau, Sant Pau Hospital, Barcelona, Spain

395. Institute for Biomedical Researhc of Barcelona (IIBB), National Spanish Research Council (CSIC)

396. Institut d'Investigacions Biomèdiques August Pi i Sunyer (IDIBAPS), Barcelona, Spain

397. Institute of Biomedicine of Valencia (IBV), CSIC, València, Spain

398. Network Center for Biomedical Research on Neurodegenerative Diseases (CIBERNED), València, Spain

399. Neurology and Genetic Mixed Unit, La Fe Helath Research Institute, València, Spain

400. Institute for Biomedical Research of Barcelona (IIBB), National Spanish Research Council (CSIC)

401. Department of Neurology, Hospital Universitari MútuaTerrassa, Fundació Docència i Recerca

MútuaTerrassa, Terrassa, Spain

402. Department of Molecular and Cell Biology, Centro Nacional de Biotecnología (CNB-CSIC), Campus Universidad Autónoma de Madrid, 28049 Madrid, Spain

403. Instituto de Física de Cantabria (IFCA-CSIC)

404. Hospital Clínic, IDIBAPS, Barcelona, Spain

405. Hospital Clínic, Barcelona, Spain 
medRxiv preprint doi: https://doi.org/10.1101/2021.11.08.21265944; this version posted February 9, 2022. The copyright holder for this preprint (which was not certified by peer review) is the author/funder, who has granted medRxiv a license to display the preprint in perpetuity.

It is made available under a CC-BY-NC-ND 4.0 International license.

406. Hospital Clínic, IDIBAPS, School of Medicine, University of Barcelona Barcelona, Spain

407. IDIBAPS, Barcelona, Spain

408. IIBB-CSIC, Barcelona, Spain

409. Servicio de Salud del Principado de Asturias, Oviedo, Spain

410. Hospital Mutua de Terrassa, Terrassa, Spain

411. Hospital Valle Hebrón, Barcelona, Spain

412. Instituto de Biomedicina y Genética Molecular (IBGM), CSIC-Universidad de Valladolid, Spain

413. Hospital Clínico Universitario de Valladolid (SACYL)

414. Department of Neurology, University Hospital of Albacete.

415. Department of Neurology

416. Research Unit, University Hospital of Albacete

417. Department of Neurology, Biomedical Research Institute Sant Pau (IIB Sant Pau), Hospital de la Santa Creu i Sant Pau, Barcelona (Spain)

418. Hospital Universitario Ramón Y Cajal, IRYCIS, Madrid, Spain

419. Institute de Biomedicine of Seville, IBiS/Hospital Universitario Virgen del Rocío/CSIC/University of Seville \& Department of Neurology, Hospital Universitario Virgen Macarena, Seville.

420. Brigham and Women's Hospital, Boston, USA

421. Harvard Medical School, Boston, MA 


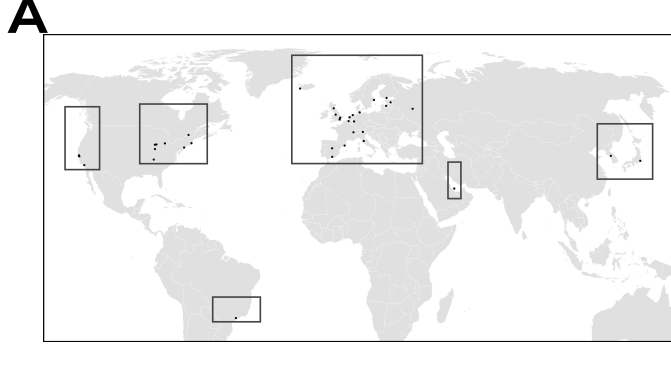

Effective

sample size

120
1,200

1,200

120,000

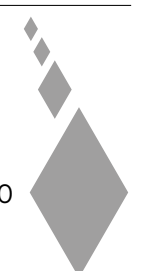

Analysis Type

Reported

SARS-CoV-2

infection

Critically ill $\bigcirc$ Hospitalised COVID-19+ COVID-19+
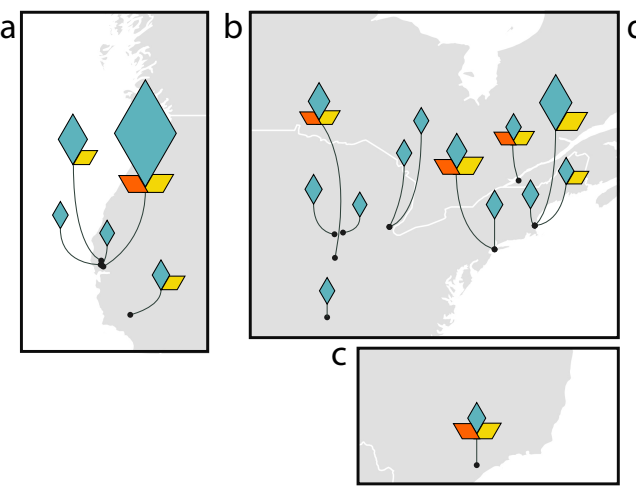

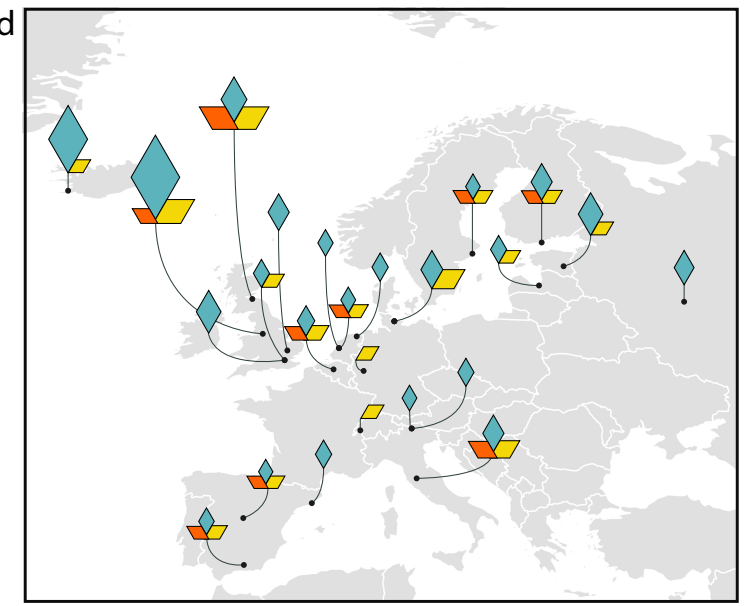

Effective sample size by genetic ancestry

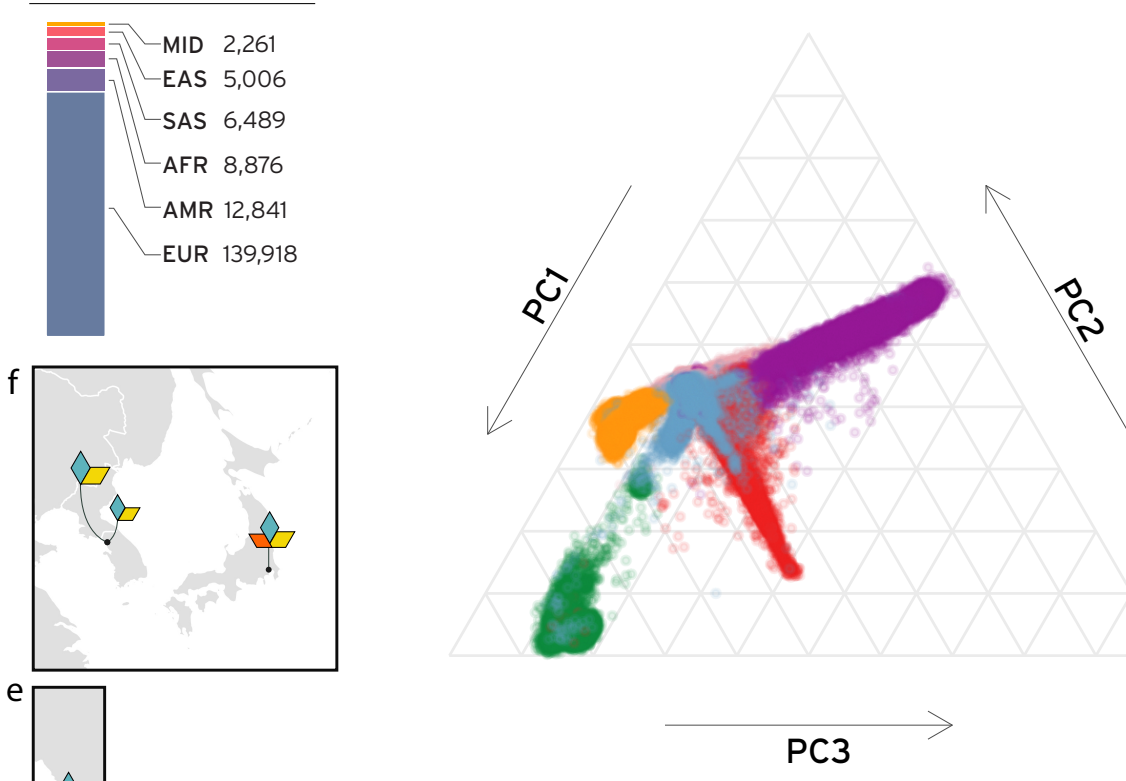

Population $\bullet$ AFR AMR $\bullet$ EAS $\odot$ EUR $\odot$ MID 
